Supporting Information for

\title{
Ruthenium Tetraoxide Oxidations of Alkanes: DFT Calculations of Barrier Heights and Kinetic Isotope Effects
}

\author{
Markus Drees, Thomas Strassner*
}

1) Coordinates of all educts, TS, intermediates
A) Educts
S 2
B) Transition states
S 5
C) Intermediates
$\mathrm{S} 18$

2) Tables for $\Delta \mathrm{E}$ (electronic energies) $\quad \mathrm{S} 31$

3) Plots of the intermediates $\quad$ S32 


\section{1) Coordinates of all educts, TS, intermediates}

A) Educts (without numbers!)

$\mathrm{RuO}_{4}$

\begin{tabular}{|c|c|c|c|c|}
\hline Center & Atomic & \multicolumn{3}{|c|}{ Coordinates (Angstroms) } \\
\hline Number & Number & $\mathrm{X}$ & $\mathrm{Y}$ & $\mathrm{Z}$ \\
\hline 1 & 44 & 0.000000 & 0.000000 & 0.000000 \\
\hline 2 & 8 & 0.979897 & 0.979897 & 0.979897 \\
\hline 3 & 8 & -0.979897 & -0.979897 & 0.979897 \\
\hline 4 & 8 & -0.979897 & 0.979897 & -0.979897 \\
\hline 5 & 8 & 0.979897 & -0.979897 & -0.979897 \\
\hline
\end{tabular}

Sum of electronic and thermal Enthalpies $=\quad-395.692088$

Sum of electronic and thermal Free Energies $=\quad-395.724698$

$\mathrm{RuO}_{4}(\mathrm{OH})$

\begin{tabular}{|c|c|c|c|c|}
\hline Center & Atomic & \multicolumn{3}{|c|}{ Coordinates (Angstroms) } \\
\hline Number & Number & $\mathrm{X}$ & $\mathrm{Y}$ & Z \\
\hline 1 & 44 & -0.112906 & -0.013635 & 0.000000 \\
\hline 2 & 8 & -0.128211 & 1.744916 & -0.001568 \\
\hline 3 & 8 & 0.752180 & -1.578735 & -0.000703 \\
\hline 4 & 8 & -1.056326 & -0.273836 & 1.424421 \\
\hline 5 & 8 & -1.059281 & -0.275586 & -1.421882 \\
\hline 6 & 8 & 1.834495 & 0.507061 & -0.000440 \\
\hline 7 & 1 & 2.225012 & -0.390613 & -0.001247 \\
\hline
\end{tabular}

Sum of electronic and thermal Enthalpies $=\quad-471.540504$

Sum of electronic and thermal Free Energies = -471.579540

Adamantane

\begin{tabular}{cccrr}
$\begin{array}{c}\text { Center } \\
\text { Number }\end{array}$ & $\begin{array}{c}\text { Atomic } \\
\text { Number }\end{array}$ & \multicolumn{2}{c}{ Coordinates } & \multicolumn{2}{c}{ (Angstroms) } \\
\hline 1 & 6 & 1.014863 & -1.053658 & 1.016242 \\
2 & 1 & 0.696184 & -1.972828 & 1.527847 \\
3 & 1 & 2.078628 & -0.910111 & 1.252548 \\
4 & 6 & 0.191642 & 0.148862 & 1.526517 \\
5 & 1 & 0.327769 & 0.254552 & 2.611221 \\
6 & 6 & 0.826251 & -1.204168 & -0.508584 \\
7 & 1 & 1.412884 & -2.059151 & -0.870750 \\
8 & 6 & -0.668880 & -1.435182 & -0.816245 \\
9 & 1 & -1.013778 & -2.359344 & -0.331403 \\
10 & 1 & -0.816695 & -1.568603 & -1.897213 \\
11 & 6 & -1.302632 & -0.082175 & 1.212268 \\
12 & 1 & -1.899412 & 0.762398 & 1.584606
\end{tabular}




\begin{tabular}{rrrrr}
13 & 1 & -1.664107 & -0.981096 & 1.731169 \\
14 & 6 & -1.496735 & -0.233977 & -0.311647 \\
15 & 1 & -2.559471 & -0.400538 & -0.533474 \\
16 & 6 & -1.015945 & 1.052077 & -1.016924 \\
17 & 1 & -1.168174 & 0.966947 & -2.102098 \\
18 & 1 & -1.611099 & 1.911842 & -0.678263 \\
19 & 6 & 0.669739 & 1.434047 & 0.816989 \\
20 & 1 & 1.728515 & 1.618857 & 1.047043 \\
21 & 1 & 0.106838 & 2.302184 & 1.187752 \\
22 & 6 & 1.302844 & 0.084937 & -1.211795 \\
23 & 1 & 2.370280 & 0.250110 & -1.008227 \\
24 & 1 & 1.198225 & -0.017210 & -2.301071 \\
25 & 6 & 0.478213 & 1.288840 & -0.708205 \\
26 & 1 & 0.817241 & 2.204375 & -1.211389 \\
\hline
\end{tabular}

Sum of electronic and thermal Enthalpies $=\quad-390.466308$ Sum of electronic and thermal Free Energies = -390.505099

Cis-decalin

\begin{tabular}{|c|c|c|c|c|}
\hline \multirow{2}{*}{$\begin{array}{l}\text { Center } \\
\text { Number }\end{array}$} & \multirow{2}{*}{$\begin{array}{l}\text { Atomic } \\
\text { Number }\end{array}$} & \multicolumn{3}{|c|}{ Coordinates (Angstroms) } \\
\hline & & $\mathrm{X}$ & $\mathrm{Y}$ & Z \\
\hline 1 & 6 & -1.541705 & -1.208007 & 0.682303 \\
\hline 2 & 6 & -2.359512 & -0.556198 & -0.444864 \\
\hline 3 & 6 & -2.232094 & 0.974047 & -0.408749 \\
\hline 4 & 6 & -0.760284 & 1.414290 & -0.449292 \\
\hline 5 & 6 & 0.060814 & 0.773847 & 0.689343 \\
\hline 6 & 6 & -0.060811 & -0.773847 & 0.689341 \\
\hline 7 & 1 & -2.786185 & 1.423678 & -1.242988 \\
\hline 8 & 1 & -2.016855 & -0.927355 & -1.420950 \\
\hline 9 & 1 & -3.413025 & -0.852081 & -0.357389 \\
\hline 10 & 1 & -1.992285 & -0.928122 & 1.646091 \\
\hline 11 & 1 & -1.607821 & -2.302715 & 0.615965 \\
\hline 12 & 1 & -0.334445 & 1.148324 & -1.426164 \\
\hline 13 & 1 & -0.690745 & 2.508123 & -0.373185 \\
\hline 14 & 1 & -0.383650 & 1.131224 & 1.631861 \\
\hline 15 & 1 & 0.383662 & -1.131229 & 1.631853 \\
\hline 16 & 1 & -2.696401 & 1.352570 & 0.514386 \\
\hline 17 & 6 & 1.541707 & 1.208012 & 0.682292 \\
\hline 18 & 1 & 1.607817 & 2.302720 & 0.615931 \\
\hline 19 & 1 & 1.992293 & 0.928149 & 1.646084 \\
\hline 20 & 6 & 2.359517 & 0.556188 & -0.444866 \\
\hline 21 & 1 & 2.016864 & 0.927338 & -1.420956 \\
\hline 22 & 1 & 3.413030 & 0.852070 & -0.357390 \\
\hline 23 & 6 & 0.760277 & -1.414281 & -0.449305 \\
\hline 24 & 1 & 0.334451 & -1.148285 & -1.426173 \\
\hline 25 & 1 & 0.690722 & -2.508115 & -0.373223 \\
\hline 26 & 6 & 2.232091 & -0.974054 & -0.408738 \\
\hline 27 & 1 & 2.786197 & -1.423702 & -1.242959 \\
\hline 28 & 1 & 2.696375 & -1.352571 & 0.514412 \\
\hline
\end{tabular}

Sum of electronic and thermal Enthalpies $=\quad-391.646488$ Sum of electronic and thermal Free Energies $=\quad-391.689287$ 
Trans-decalin

\begin{tabular}{ccrrr}
$\begin{array}{c}\text { Center } \\
\text { Number }\end{array}$ & $\begin{array}{c}\text { Atomic } \\
\text { Number }\end{array}$ & \multicolumn{1}{c}{ Coordinates } & \multicolumn{1}{c}{ (Angstroms) } \\
\hline 1 & 6 & 0.227960 & 1.461520 & 1.277186 \\
2 & 6 & -0.232164 & 0.737787 & 0.000000 \\
3 & 6 & 0.232164 & -0.737787 & 0.000000 \\
4 & 6 & -0.227960 & -1.461520 & 1.277186 \\
5 & 6 & 0.227960 & -0.733561 & 2.550702 \\
6 & 6 & -0.227960 & 0.733561 & 2.550702 \\
7 & 1 & -1.336339 & 0.730256 & 0.000000 \\
8 & 1 & 1.326759 & 1.531700 & 1.272284 \\
9 & 1 & -0.147286 & 2.494186 & 1.274692 \\
10 & 1 & -1.326759 & -1.531700 & 1.272284 \\
11 & 1 & 0.147286 & -2.494186 & 1.274692 \\
12 & 1 & -0.152640 & -1.251131 & 3.440815 \\
13 & 1 & 1.325763 & -0.770547 & 2.614165 \\
14 & 1 & -1.325763 & 0.770547 & 2.614165 \\
15 & 1 & 0.152640 & 1.251131 & 3.440815 \\
16 & 1 & 1.336339 & -0.730256 & 0.000000 \\
17 & 6 & -0.227960 & -1.461520 & -1.277186 \\
18 & 1 & -1.326759 & -1.531700 & -1.272284 \\
19 & 1 & 0.147286 & -2.494186 & -1.274692 \\
20 & 6 & 0.227960 & 1.461520 & -1.277186 \\
21 & 1 & -0.147286 & 2.494186 & -1.274692 \\
22 & 1 & 1.326759 & 1.531700 & -1.272284 \\
23 & 6 & -0.227960 & 0.733561 & -2.550702 \\
24 & 1 & 0.152640 & 1.251131 & -3.440815 \\
25 & 1 & -1.325763 & 0.770547 & -2.614165 \\
26 & 6 & 0.227960 & -0.733561 & -2.550702 \\
27 & 1 & -0.152640 & -1.251131 & -3.440815 \\
28 & 1 & -325763 & -0.770547 & -2.614165 \\
\hline
\end{tabular}

Sum of electronic and thermal Enthalpies $=\quad-391.651964$

Sum of electronic and thermal Free Energies = -391.694268

Methane

\begin{tabular}{ccrrr}
$\begin{array}{c}\text { Center } \\
\text { Number }\end{array}$ & Atomic & \multicolumn{2}{c}{ Coordinates } & (Angstroms) \\
\hline 1 & Number & \multicolumn{1}{c}{$\mathrm{X}$} & \multicolumn{1}{c}{$\mathrm{Y}$} & \multicolumn{1}{c}{ Z } \\
\hline 2 & 6 & 0.000000 & 0.000000 & 0.00000 \\
3 & 1 & 0.631397 & 0.631397 & 0.631397 \\
4 & 1 & -0.631397 & -0.631397 & 0.631397 \\
5 & 1 & -0.631397 & 0.631397 & -0.631397 \\
\hline
\end{tabular}

Sum of electronic and thermal Enthalpies $=\quad-40.468641$

Sum of electronic and thermal Free Energies $=\quad-40.489773$ 
B) Transition states

Oxidation with $\mathrm{RuO}_{4}$

TSla (adamantane, tertiary)

\begin{tabular}{|c|c|c|c|c|}
\hline \multirow{2}{*}{$\begin{array}{l}\text { Center } \\
\text { Number }\end{array}$} & Atomic & \multicolumn{3}{|c|}{ Coordinates (Angstroms) } \\
\hline & Number & $\mathrm{X}$ & $\mathrm{Y}$ & Z \\
\hline 1 & 6 & 3.605517 & -1.262300 & 0.580210 \\
\hline 2 & 1 & 4.695529 & -1.286937 & 0.445894 \\
\hline 3 & 1 & 3.334426 & -2.163753 & 1.145839 \\
\hline 4 & 6 & 2.905592 & -1.262675 & -0.792724 \\
\hline 5 & 1 & 3.177435 & -2.163933 & -1.356731 \\
\hline 6 & 6 & 3.203025 & 0.000403 & 1.365759 \\
\hline 7 & 1 & 3.680297 & 0.000684 & 2.354344 \\
\hline 8 & 6 & 3.605413 & 1.262715 & 0.579495 \\
\hline 9 & 1 & 4.695421 & 1.287349 & 0.445149 \\
\hline 10 & 1 & 3.334261 & 2.164451 & 1.144641 \\
\hline 11 & 6 & 3.304326 & -0.000405 & -1.584162 \\
\hline 12 & 1 & 2.817442 & -0.000697 & -2.568134 \\
\hline 13 & 1 & 4.387899 & -0.000396 & -1.764984 \\
\hline 14 & 6 & 2.905467 & 1.262257 & -0.793434 \\
\hline 15 & 1 & 3.177237 & 2.163227 & -1.357933 \\
\hline 16 & 6 & 1.354324 & 1.261775 & -0.587293 \\
\hline 17 & 1 & 1.051388 & 2.157372 & -0.031155 \\
\hline 18 & 1 & 0.845663 & 1.275894 & -1.554754 \\
\hline 19 & 6 & 1.354453 & -1.262136 & -0.586649 \\
\hline 20 & 1 & 1.051488 & -2.157446 & -0.030071 \\
\hline 21 & 1 & 0.845854 & -1.276716 & -1.554134 \\
\hline 22 & 6 & 1.654424 & 0.000394 & 1.572788 \\
\hline 23 & 1 & 1.351901 & -0.885405 & 2.142868 \\
\hline 24 & 1 & 1.351861 & 0.886477 & 2.142406 \\
\hline 25 & 6 & 1.026197 & 0.000015 & 0.181826 \\
\hline 26 & 1 & -0.276417 & -0.000194 & 0.624932 \\
\hline 27 & 8 & -1.298967 & -0.000162 & 1.270212 \\
\hline 28 & 8 & -3.435781 & -1.461457 & -0.027083 \\
\hline 29 & 8 & -3.435216 & 1.461816 & -0.027168 \\
\hline 30 & 8 & -1.330830 & -0.000302 & -1.226951 \\
\hline 31 & 44 & -2.568813 & 0.000014 & 0.005565 \\
\hline
\end{tabular}

Sum of electronic and thermal Enthalpies =

$-786.136212$

Sum of electronic and thermal Free Energies = $-786.192161$ NIMAG $=1, \quad-1050.7887 \mathrm{~cm}^{-1}$ 
TS1b (adamantane, secondary)

\begin{tabular}{|c|c|c|c|c|}
\hline Center & Atomic & \multicolumn{3}{|c|}{ Coordinates (Angstroms) } \\
\hline Number & Number & $\mathrm{X}$ & Y & $\mathrm{z}$ \\
\hline 1 & 6 & -0.900009 & -0.612482 & 0.521352 \\
\hline 2 & 1 & -0.589791 & -1.277994 & 1.322567 \\
\hline 3 & 1 & 0.197956 & -0.353156 & -0.293267 \\
\hline 4 & 6 & -1.471809 & 0.708798 & 0.986449 \\
\hline 5 & 1 & -0.792265 & 1.187619 & 1.698002 \\
\hline 6 & 6 & -1.765782 & -1.293121 & -0.533644 \\
\hline 7 & 1 & -1.296257 & -2.219936 & -0.882639 \\
\hline 8 & 6 & -3.099418 & -1.638711 & 0.209782 \\
\hline 9 & 1 & -2.901584 & -2.322564 & 1.044830 \\
\hline 10 & 1 & -3.757489 & -2.167043 & -0.493694 \\
\hline 11 & 6 & -2.815345 & 0.360650 & 1.706779 \\
\hline 12 & 1 & -3.263462 & 1.290836 & 2.081103 \\
\hline 13 & 1 & -2.620536 & -0.278889 & 2.576992 \\
\hline 14 & 6 & -3.761295 & -0.342032 & 0.714798 \\
\hline 15 & 1 & -4.701639 & -0.592330 & 1.224215 \\
\hline 16 & 6 & -4.048719 & 0.597323 & -0.475748 \\
\hline 17 & 1 & -4.745938 & 0.116657 & -1.175364 \\
\hline 18 & 1 & -4.536822 & 1.514236 & -0.118457 \\
\hline 19 & 6 & -1.767537 & 1.641264 & -0.208773 \\
\hline 20 & 1 & -0.832374 & 1.911708 & -0.714796 \\
\hline 21 & 1 & -2.213261 & 2.574322 & 0.159853 \\
\hline 22 & 6 & -2.074597 & -0.353583 & -1.716676 \\
\hline 23 & 1 & -1.153533 & -0.126899 & -2.265826 \\
\hline 24 & 1 & -2.748277 & -0.863362 & -2.418040 \\
\hline 25 & 6 & -2.728373 & 0.943615 & -1.195973 \\
\hline 26 & 1 & -2.933316 & 1.613190 & -2.040784 \\
\hline 27 & 8 & 1.086394 & -0.170628 & -1.076901 \\
\hline 28 & 8 & 1.495032 & -0.237581 & 1.380445 \\
\hline 29 & 8 & 3.615307 & -1.275418 & -0.177456 \\
\hline 30 & 8 & 3.120359 & 1.602335 & -0.033557 \\
\hline 31 & 44 & 2.521572 & 0.013037 & -0.011250 \\
\hline
\end{tabular}

Sum of electronic and thermal Enthalpies =

$-786.132220$

Sum of electronic and thermal Free Energies =

$-786.187465$ 
TS2a (cis-decalin, tertiary)

\begin{tabular}{|c|c|c|c|c|}
\hline \multirow{2}{*}{$\begin{array}{l}\text { Center } \\
\text { Number }\end{array}$} & \multirow{2}{*}{$\begin{array}{l}\text { Atomic } \\
\text { Number }\end{array}$} & \multicolumn{3}{|c|}{ Coordinates (Angstroms) } \\
\hline & & $\mathrm{X}$ & Y & Z \\
\hline 1 & 6 & 2.041991 & 1.702228 & -1.243192 \\
\hline 2 & 6 & 2.275002 & 2.592798 & -0.013141 \\
\hline 3 & 6 & 1.326187 & 2.212997 & 1.132093 \\
\hline 4 & 6 & 1.461022 & 0.725564 & 1.493976 \\
\hline 5 & 6 & 1.298596 & -0.203661 & 0.303280 \\
\hline 6 & 6 & 2.117928 & 0.192428 & -0.933486 \\
\hline 7 & 1 & 1.529155 & 2.824096 & 2.019563 \\
\hline 8 & 1 & 3.315324 & 2.503305 & 0.330244 \\
\hline 9 & 1 & 2.132242 & 3.644282 & -0.290531 \\
\hline 10 & 1 & 1.048045 & 1.920317 & -1.653668 \\
\hline 11 & 1 & 2.765030 & 1.941960 & -2.033391 \\
\hline 12 & 1 & 2.478701 & 0.559139 & 1.891350 \\
\hline 13 & 1 & 0.764629 & 0.443880 & 2.286977 \\
\hline 14 & 1 & 0.067166 & 0.039560 & -0.285039 \\
\hline 15 & 1 & 1.716008 & -0.356887 & -1.796161 \\
\hline 16 & 1 & 0.288064 & 2.420405 & 0.838884 \\
\hline 17 & 6 & 1.316281 & -1.693067 & 0.588146 \\
\hline 18 & 1 & 0.735773 & -1.916727 & 1.487088 \\
\hline 19 & 1 & 0.847134 & -2.231952 & -0.246246 \\
\hline 20 & 6 & 2.774221 & -2.203967 & 0.737016 \\
\hline 21 & 1 & 3.217607 & -1.779325 & 1.647866 \\
\hline 22 & 1 & 2.752100 & -3.291557 & 0.875977 \\
\hline 23 & 6 & 3.580040 & -0.316784 & -0.744458 \\
\hline 24 & 1 & 4.061996 & 0.220770 & 0.082227 \\
\hline 25 & 1 & 4.141944 & -0.065594 & -1.653332 \\
\hline 26 & 6 & 3.626337 & -1.826079 & -0.478978 \\
\hline 27 & 1 & 4.664655 & -2.148769 & -0.328778 \\
\hline 28 & 1 & 3.256746 & -2.362465 & -1.364744 \\
\hline 29 & 8 & -0.846109 & 0.190518 & -1.072988 \\
\hline 30 & 44 & -2.322670 & -0.124239 & -0.109241 \\
\hline 31 & 8 & -3.268949 & 1.274525 & 0.082628 \\
\hline 32 & 8 & -1.352063 & -0.400610 & 1.314639 \\
\hline 33 & 8 & -3.094185 & -1.563516 & -0.580431 \\
\hline
\end{tabular}

Sum of electronic and thermal Enthalpies $=\quad-787.316507$ Sum of electronic and thermal Free Energies $=\quad-787.375616$ NIMAG $=1, \quad-1089.5782 \mathrm{~cm}^{-1}$ 
TS2b (cis-decalin, secondary)

\begin{tabular}{|c|c|c|c|c|}
\hline \multirow{2}{*}{$\begin{array}{l}\text { Center } \\
\text { Number }\end{array}$} & \multirow{2}{*}{$\begin{array}{l}\text { Atomic } \\
\text { Number }\end{array}$} & \multicolumn{3}{|c|}{ Coordinates (Angstroms) } \\
\hline & & $\mathrm{X}$ & $\mathrm{Y}$ & $\mathrm{Z}$ \\
\hline 1 & 6 & 4.260447 & -0.525740 & 0.941844 \\
\hline 2 & 6 & 4.442083 & -1.420812 & -0.293281 \\
\hline 3 & 6 & 3.144442 & -2.169801 & -0.626790 \\
\hline 4 & 6 & 1.959019 & -1.206613 & -0.799295 \\
\hline 5 & 6 & 1.764213 & -0.327854 & 0.452142 \\
\hline 6 & 6 & 3.081279 & 0.462630 & 0.814218 \\
\hline 7 & 1 & 3.271101 & -2.768566 & -1.536977 \\
\hline 8 & 1 & 4.747424 & -0.814417 & -1.157106 \\
\hline 9 & 1 & 5.257229 & -2.133094 & -0.114777 \\
\hline 10 & 1 & 4.087460 & -1.163652 & 1.820000 \\
\hline 11 & 1 & 5.179799 & 0.040152 & 1.144872 \\
\hline 12 & 1 & 2.124236 & -0.582814 & -1.687210 \\
\hline 13 & 1 & 1.040913 & -1.773875 & -0.985921 \\
\hline 14 & 1 & 1.555020 & -0.996458 & 1.298874 \\
\hline 15 & 1 & 2.920495 & 0.921713 & 1.800329 \\
\hline 16 & 1 & 2.916593 & -2.878235 & 0.183288 \\
\hline 17 & 6 & 0.663287 & 0.721973 & 0.388034 \\
\hline 18 & 1 & -0.352661 & -0.057428 & -0.149652 \\
\hline 19 & 1 & 0.410277 & 1.091420 & 1.377443 \\
\hline 20 & 6 & 0.841081 & 1.834571 & -0.616184 \\
\hline 21 & 1 & 0.900058 & 1.442072 & -1.638335 \\
\hline 22 & 1 & -0.005243 & 2.526986 & -0.574136 \\
\hline 23 & 6 & 3.345183 & 1.608654 & -0.181053 \\
\hline 24 & 1 & 3.564220 & 1.209092 & -1.179107 \\
\hline 25 & 1 & 4.245174 & 2.152640 & 0.135652 \\
\hline 26 & 6 & 2.159927 & 2.579441 & -0.271388 \\
\hline 27 & 1 & 2.343769 & 3.351331 & -1.029019 \\
\hline 28 & 1 & 2.033133 & 3.098601 & 0.688264 \\
\hline 29 & 8 & -1.170823 & -0.734186 & -0.707297 \\
\hline 30 & 44 & -2.713907 & -0.179166 & 0.025685 \\
\hline 31 & 8 & -3.675914 & 0.659172 & -1.095064 \\
\hline 32 & 8 & -1.818186 & 0.942629 & 1.022380 \\
\hline 33 & 8 & -3.434186 & -1.382722 & 0.982971 \\
\hline
\end{tabular}

Sum of electronic and thermal Enthalpies $=\quad-787.312249$ Sum of electronic and thermal Free Energies $=\quad-787.372434$ NIMAG $=1, \quad-1033.3727 \mathrm{~cm}^{-1}$ 
TS3a (trans-decalin, tertiary)

\begin{tabular}{|c|c|c|c|c|}
\hline \multirow{2}{*}{$\begin{array}{l}\text { Center } \\
\text { Number }\end{array}$} & \multirow{2}{*}{$\begin{array}{l}\text { Atomic } \\
\text { Number }\end{array}$} & \multicolumn{3}{|c|}{ Coordinates (Angstroms) } \\
\hline & & $\mathrm{X}$ & $\mathrm{Y}$ & Z \\
\hline 1 & 6 & -1.470341 & 1.291135 & 1.515846 \\
\hline 2 & 6 & -1.487058 & 0.000153 & 0.718539 \\
\hline 3 & 6 & -2.493810 & -0.000129 & -0.440899 \\
\hline 4 & 6 & -2.436714 & 1.284038 & -1.289638 \\
\hline 5 & 6 & -2.484828 & 2.557444 & -0.433041 \\
\hline 6 & 6 & -1.388420 & 2.552806 & 0.642381 \\
\hline 7 & 1 & -0.328844 & 0.000489 & -0.092199 \\
\hline 8 & 1 & -2.426224 & 1.313872 & 2.072210 \\
\hline 9 & 1 & -0.671290 & 1.266939 & 2.260622 \\
\hline 10 & 1 & -1.515655 & 1.275787 & -1.886121 \\
\hline 11 & 1 & -3.271314 & 1.272917 & -2.002194 \\
\hline 12 & 1 & -2.383486 & 3.442023 & -1.073483 \\
\hline 13 & 1 & -3.468658 & 2.634778 & 0.053495 \\
\hline 14 & 1 & -0.401057 & 2.601589 & 0.164509 \\
\hline 15 & 1 & -1.471741 & 3.441334 & 1.279320 \\
\hline 16 & 1 & -3.470100 & -0.000578 & 0.086560 \\
\hline 17 & 6 & -2.435407 & -1.284337 & -1.289512 \\
\hline 18 & 1 & -1.513632 & -1.275695 & -1.884917 \\
\hline 19 & 1 & -3.269148 & -1.273621 & -2.003072 \\
\hline 20 & 6 & -1.472528 & -1.290185 & 1.516922 \\
\hline 21 & 1 & -0.675096 & -1.265938 & 2.263364 \\
\hline 22 & 1 & -2.429754 & -1.312280 & 2.070993 \\
\hline 23 & 6 & -1.389202 & -2.552382 & 0.644282 \\
\hline 24 & 1 & -1.473502 & -3.440575 & 1.281561 \\
\hline 25 & 1 & -0.401018 & -2.601321 & 0.168122 \\
\hline 26 & 6 & -2.483985 & -2.557679 & -0.432828 \\
\hline 27 & 1 & -2.381213 & -3.442256 & -1.073048 \\
\hline 28 & 1 & -3.468477 & -2.635520 & 0.052267 \\
\hline 29 & 8 & 0.489383 & 0.003983 & -0.977587 \\
\hline 30 & 8 & 2.920759 & 1.459273 & -0.361123 \\
\hline 31 & 8 & 2.920276 & -1.457567 & -0.373808 \\
\hline 32 & 8 & 1.272646 & -0.006624 & 1.406830 \\
\hline 33 & 44 & 2.078397 & 0.000008 & -0.141018 \\
\hline
\end{tabular}

Sum of electronic and thermal Enthalpies =

$-787.318448$

Sum of electronic and thermal Free Energies = NIMAG $=1, \quad-1085.4114 \mathrm{~cm}^{-1}$ 
TS3b (trans-decalin, secondary)

\begin{tabular}{|c|c|c|c|c|}
\hline Center & Atomic & \multicolumn{3}{|c|}{ Coordinates (Angstroms) } \\
\hline Number & Number & $\mathrm{X}$ & Y & $\mathrm{Z}$ \\
\hline 1 & 6 & 1.745533 & -1.627008 & 0.310260 \\
\hline 2 & 6 & 1.812800 & -0.168347 & -0.171845 \\
\hline 3 & 6 & 3.183708 & 0.490075 & 0.215213 \\
\hline 4 & 6 & 4.343179 & -0.364357 & -0.329511 \\
\hline 5 & 6 & 4.270305 & -1.823088 & 0.142329 \\
\hline 6 & 6 & 2.921071 & -2.459731 & -0.220807 \\
\hline 7 & 1 & 1.752739 & -0.155216 & -1.271024 \\
\hline 8 & 1 & 1.752197 & -1.640461 & 1.410383 \\
\hline 9 & 1 & 0.793891 & -2.071271 & -0.005373 \\
\hline 10 & 1 & 4.323332 & -0.332582 & -1.429082 \\
\hline 11 & 1 & 5.295053 & 0.088887 & -0.021134 \\
\hline 12 & 1 & 5.094453 & -2.400281 & -0.294923 \\
\hline 13 & 1 & 4.407793 & -1.858955 & 1.232994 \\
\hline 14 & 1 & 2.840275 & -2.543063 & -1.314411 \\
\hline 15 & 1 & 2.862113 & -3.481205 & 0.174201 \\
\hline 16 & 1 & 3.246116 & 0.487204 & 1.315428 \\
\hline 17 & 6 & 3.245623 & 1.948036 & -0.264442 \\
\hline 18 & 1 & 3.229209 & 1.967687 & -1.364340 \\
\hline 19 & 1 & 4.202984 & 2.391680 & 0.039958 \\
\hline 20 & 6 & 0.714550 & 0.715175 & 0.395610 \\
\hline 21 & 1 & -0.320997 & 0.134396 & -0.287294 \\
\hline 22 & 1 & 0.606966 & 0.608126 & 1.472666 \\
\hline 23 & 6 & 0.715330 & 2.149064 & -0.075463 \\
\hline 24 & 1 & -0.101388 & 2.709699 & 0.388986 \\
\hline 25 & 1 & 0.575185 & 2.192550 & -1.163830 \\
\hline 26 & 6 & 2.084336 & 2.787237 & 0.281013 \\
\hline 27 & 1 & 2.117244 & 3.807826 & -0.119824 \\
\hline 28 & 1 & 2.168258 & 2.871148 & 1.372933 \\
\hline 29 & 8 & -1.155889 & -0.347695 & -1.019934 \\
\hline 30 & 8 & -1.699961 & 0.531619 & 1.245290 \\
\hline 31 & 8 & -3.259207 & -1.695181 & 0.445546 \\
\hline 32 & 8 & -3.715073 & 0.954928 & -0.699048 \\
\hline 33 & 44 & -2.645977 & -0.186543 & -0.036484 \\
\hline
\end{tabular}

Sum of electronic and thermal Enthalpies $=\quad-787.318920$ Sum of electronic and thermal Free Energies $=\quad-787.378657$ NIMAG $=1, \quad-1052.3908 \mathrm{~cm}^{-1}$ 
TS4 (Methane)

\begin{tabular}{|c|c|c|c|c|}
\hline Center & Atomic & \multicolumn{3}{|c|}{ Coordinates (Angstroms) } \\
\hline Number & Number & $\mathrm{X}$ & Y & Z \\
\hline 1 & 6 & -2.880798 & -0.000003 & -0.096460 \\
\hline 2 & 1 & -3.021967 & -0.911025 & -0.667496 \\
\hline 3 & 1 & -1.755040 & 0.000015 & 0.709348 \\
\hline 4 & 1 & -3.021834 & 0.911006 & -0.667546 \\
\hline 5 & 1 & -3.438534 & 0.000049 & 0.849363 \\
\hline 6 & 44 & 0.460306 & 0.000000 & -0.001279 \\
\hline 7 & 8 & -0.738273 & -0.000017 & 1.331443 \\
\hline 8 & 8 & -0.869436 & 0.000001 & -1.134498 \\
\hline 9 & 8 & 1.320637 & 1.458082 & -0.072755 \\
\hline 10 & 8 & 1.320661 & -1.458068 & -0.072771 \\
\hline
\end{tabular}

Sum of electronic and thermal Enthalpies $=\quad-436.118775$ Sum of electronic and thermal Free Energies $=\quad-436.159304$ NIMAG $=1, \quad-1135.9843 \mathrm{~cm}^{-1}$

Oxidations with $\mathrm{RuO}_{4}(\mathrm{OH})^{-}$

TS8 (Methane)

\begin{tabular}{ccrrr}
$\begin{array}{c}\text { Center } \\
\text { Number }\end{array}$ & Atomic & \multicolumn{2}{c}{ Coordinates } & \multicolumn{1}{c}{ (Angstroms) } \\
\hline 1 & Number & $\mathrm{X}$ & \multicolumn{1}{c}{$\mathrm{Z}$} \\
2 & 6 & 3.018303 & 0.507431 & -0.062986 \\
3 & 1 & 3.035938 & 1.284367 & 0.695818 \\
4 & 1 & 2.016980 & -0.448944 & 0.124752 \\
5 & 1 & 3.060246 & 0.897929 & -1.075748 \\
6 & 1 & 3.777916 & -0.265180 & 0.116300 \\
7 & 4 & -0.307767 & -0.112701 & 0.004373 \\
8 & 8 & 1.179036 & -1.224275 & 0.266487 \\
9 & 8 & 0.804738 & 1.221200 & -0.237149 \\
10 & 8 & -0.837051 & -0.851621 & -1.451492 \\
11 & 8 & -2.032463 & 1.014149 & -0.250191 \\
12 & 8 & -0.888352 & -0.259682 & 1.630080 \\
\hline
\end{tabular}

Sum of electronic and thermal Enthalpies $=\quad-511.979790$ Sum of electronic and thermal Free Energies = $\quad-512.024059$ NIMAG $=1, \quad-911.9374 \mathrm{~cm}^{-1}$ 
TS5a (admantane, tertiary)

\begin{tabular}{|c|c|c|c|c|}
\hline Center & Atomic & \multicolumn{3}{|c|}{ Coordinates (Angstroms) } \\
\hline Number & Number & $\mathrm{X}$ & $\mathrm{Y}$ & Z \\
\hline 1 & 6 & -3.824819 & -0.668888 & 1.165802 \\
\hline 2 & 1 & -4.908490 & -0.481969 & 1.217620 \\
\hline 3 & 1 & -3.584487 & -1.384338 & 1.965105 \\
\hline 4 & 6 & -3.045583 & 0.644565 & 1.381115 \\
\hline 5 & 1 & -3.306374 & 1.073703 & 2.359701 \\
\hline 6 & 6 & -3.456019 & -1.269729 & -0.206838 \\
\hline 7 & 1 & -4.001249 & -2.213163 & -0.359868 \\
\hline 8 & 6 & -3.821391 & -0.268753 & -1.322445 \\
\hline 9 & 1 & -4.904998 & -0.074573 & -1.316354 \\
\hline 10 & 1 & -3.578453 & -0.697227 & -2.305283 \\
\hline 11 & 6 & -3.408717 & 1.644775 & 0.261924 \\
\hline 12 & 1 & -2.868582 & 2.589221 & 0.415490 \\
\hline 13 & 1 & -4.483834 & 1.879093 & 0.298671 \\
\hline 14 & 6 & -3.043051 & 1.046871 & -1.114470 \\
\hline 15 & 1 & -3.303503 & 1.761371 & -1.909167 \\
\hline 16 & 6 & -1.514527 & 0.762620 & -1.153419 \\
\hline 17 & 1 & -1.235722 & 0.342257 & -2.129359 \\
\hline 18 & 1 & -0.950524 & 1.690951 & -1.024363 \\
\hline 19 & 6 & -1.517115 & 0.360968 & 1.331521 \\
\hline 20 & 1 & -1.240549 & -0.345394 & 2.126167 \\
\hline 21 & 1 & -0.953843 & 1.282348 & 1.504240 \\
\hline 22 & 6 & -1.928635 & -1.543118 & -0.250768 \\
\hline 23 & 1 & -1.650248 & -2.266728 & 0.525720 \\
\hline 24 & 1 & -1.650764 & -1.984452 & -1.216205 \\
\hline 25 & 6 & -1.185418 & -0.216288 & -0.036483 \\
\hline 26 & 1 & 0.082479 & -0.780979 & -0.143968 \\
\hline 27 & 8 & 1.042197 & -1.417812 & -0.270173 \\
\hline 28 & 8 & 3.903206 & 1.241599 & 0.263322 \\
\hline 29 & 8 & 2.966868 & -0.180203 & -1.622534 \\
\hline 30 & 8 & 1.062594 & 1.067395 & 0.205809 \\
\hline 31 & 44 & 2.352182 & -0.103737 & -0.002306 \\
\hline 32 & 8 & 2.950658 & -0.747232 & 1.474232 \\
\hline 33 & 1 & 4.110614 & 1.506207 & -0.647584 \\
\hline
\end{tabular}

Sum of electronic and thermal Enthalpies $=\quad-861.988259$ Sum of electronic and thermal Free Energies $=\quad-862.047209$ NIMAG $=1, \quad-887.8188 \mathrm{~cm}^{-1}$ 
TS5b (adamantane, secondary)

\begin{tabular}{|c|c|c|c|c|}
\hline Center & Atomic & \multicolumn{3}{|c|}{ Coordinates (Angstroms) } \\
\hline Number & Number & $\mathrm{X}$ & Y & Z \\
\hline 1 & 6 & -1.213389 & -0.995862 & 0.147860 \\
\hline 2 & 1 & -1.257524 & -2.088077 & 0.265989 \\
\hline 3 & 1 & 0.206910 & -1.186529 & 0.122522 \\
\hline 4 & 6 & -1.889812 & -0.312016 & 1.325927 \\
\hline 5 & 1 & -1.390316 & -0.584011 & 2.264397 \\
\hline 6 & 6 & -1.827429 & -0.576596 & -1.178310 \\
\hline 7 & 1 & -1.280118 & -1.035339 & -2.011239 \\
\hline 8 & 6 & -3.294057 & -1.104554 & -1.167312 \\
\hline 9 & 1 & -3.301967 & -2.200608 & -1.087825 \\
\hline 10 & 1 & -3.791298 & -0.845794 & -2.114653 \\
\hline 11 & 6 & -3.357170 & -0.842122 & 1.347643 \\
\hline 12 & 1 & -3.900729 & -0.398027 & 2.195624 \\
\hline 13 & 1 & -3.366731 & -1.931341 & 1.494400 \\
\hline 14 & 6 & -4.057483 & -0.476520 & 0.019664 \\
\hline 15 & 1 & -5.089787 & -0.857350 & 0.034239 \\
\hline 16 & 6 & -4.073176 & 1.058530 & -0.144185 \\
\hline 17 & 1 & -4.590074 & 1.329774 & -1.077016 \\
\hline 18 & 1 & -4.638864 & 1.518013 & 0.680371 \\
\hline 19 & 6 & -1.928175 & 1.221137 & 1.168109 \\
\hline 20 & 1 & -0.908104 & 1.610710 & 1.181829 \\
\hline 21 & 1 & -2.479126 & 1.662932 & 2.012941 \\
\hline 22 & 6 & -1.853367 & 0.956677 & -1.338092 \\
\hline 23 & 1 & -0.827801 & 1.332821 & -1.364060 \\
\hline 24 & 1 & -2.342329 & 1.219709 & -2.288677 \\
\hline 25 & 6 & -2.623916 & 1.589922 & -0.159551 \\
\hline 26 & 1 & -2.635571 & 2.682946 & -0.275198 \\
\hline 27 & 8 & 1.235875 & -1.648106 & 0.054723 \\
\hline 28 & 8 & 0.847192 & 0.868101 & 0.088837 \\
\hline 29 & 8 & 3.059469 & -0.391626 & 1.520862 \\
\hline 30 & 8 & 2.860207 & -0.350581 & -1.621648 \\
\hline 31 & 44 & 2.306252 & -0.106945 & -0.014148 \\
\hline 32 & 8 & 3.618364 & 1.490873 & -0.090756 \\
\hline 33 & 1 & 3.857313 & 1.614871 & 0.842202 \\
\hline
\end{tabular}

Sum of electronic and thermal Enthalpies $=\quad-861.980945$ Sum of electronic and thermal Free Energies $=\quad-862.040309$ NIMAG $=1, \quad-704.2698 \mathrm{~cm}^{-1}$ 
TS6a (cis-decalin, tertiary)

\begin{tabular}{|c|c|c|c|c|}
\hline Center & Atomic & \multicolumn{3}{|c|}{ Coordinates (Angstroms) } \\
\hline Number & Number & $\mathrm{X}$ & $\mathrm{Y}$ & $\mathrm{Z}$ \\
\hline 1 & 6 & 2.293950 & 1.700347 & -1.169812 \\
\hline 2 & 6 & 2.477979 & 2.568460 & 0.085019 \\
\hline 3 & 6 & 1.478872 & 2.167809 & 1.180260 \\
\hline 4 & 6 & 1.605068 & 0.674353 & 1.518761 \\
\hline 5 & 6 & 1.442347 & -0.216827 & 0.295534 \\
\hline 6 & 6 & 2.352348 & 0.185035 & -0.883919 \\
\hline 7 & 1 & 1.640488 & 2.771544 & 2.084139 \\
\hline 8 & 1 & 3.503736 & 2.467143 & 0.472941 \\
\hline 9 & 1 & 2.352104 & 3.627732 & -0.179074 \\
\hline 10 & 1 & 1.309888 & 1.915310 & -1.601581 \\
\hline 11 & 1 & 3.046848 & 1.960472 & -1.928852 \\
\hline 12 & 1 & 2.608389 & 0.507106 & 1.957291 \\
\hline 13 & 1 & 0.871672 & 0.389476 & 2.276908 \\
\hline 14 & 1 & 0.253504 & 0.070937 & -0.397029 \\
\hline 15 & 1 & 1.974596 & -0.331909 & -1.777892 \\
\hline 16 & 1 & 0.455551 & 2.367155 & 0.838182 \\
\hline 17 & 6 & 1.467121 & -1.715100 & 0.553623 \\
\hline 18 & 1 & 0.847477 & -1.953319 & 1.422595 \\
\hline 19 & 1 & 1.013285 & -2.230396 & -0.305140 \\
\hline 20 & 6 & 2.906021 & -2.249802 & 0.745458 \\
\hline 21 & 1 & 3.326396 & -1.849884 & 1.680119 \\
\hline 22 & 1 & 2.881052 & -3.342762 & 0.858668 \\
\hline 23 & 6 & 3.795248 & -0.335837 & -0.655853 \\
\hline 24 & 1 & 4.251623 & 0.168450 & 0.207522 \\
\hline 25 & 1 & 4.410666 & -0.074188 & -1.529452 \\
\hline 26 & 6 & 3.815246 & -1.854074 & -0.425887 \\
\hline 27 & 1 & 4.843962 & -2.200840 & -0.249933 \\
\hline 28 & 1 & 3.465753 & -2.359187 & -1.339166 \\
\hline 29 & 8 & -0.583174 & 0.274718 & -1.174862 \\
\hline 30 & 44 & -2.127311 & -0.058443 & -0.163138 \\
\hline 31 & 8 & -2.698043 & 1.563375 & -0.192032 \\
\hline 32 & 8 & -1.102795 & -0.426435 & 1.212123 \\
\hline 33 & 8 & -3.924308 & -0.389088 & 0.800813 \\
\hline 34 & 8 & -2.586072 & -1.467076 & -1.067258 \\
\hline 35 & 1 & -4.105356 & -1.321497 & 0.598444 \\
\hline
\end{tabular}

Sum of electronic and thermal Enthalpies $=\quad-863.170302$ Sum of electronic and thermal Free Energies = -863.232640 NIMAG $=1,-908.9523 \mathrm{~cm}^{-1}$ 
TS6b (cis-decalin, secondary)

\begin{tabular}{|c|c|c|c|c|}
\hline Center & Atomic & \multicolumn{3}{|c|}{ Coordinates (Angstroms) } \\
\hline Number & Number & $\mathrm{X}$ & Y & $\mathrm{Z}$ \\
\hline 1 & 6 & 0.934754 & 1.080489 & 0.597975 \\
\hline 2 & 6 & 1.146732 & 2.023858 & -0.568493 \\
\hline 3 & 6 & 2.605998 & 2.534469 & -0.555275 \\
\hline 4 & 6 & 3.599206 & 1.360725 & -0.552127 \\
\hline 5 & 6 & 3.346419 & 0.395834 & 0.625707 \\
\hline 6 & 6 & 1.868426 & -0.123326 & 0.640621 \\
\hline 7 & 1 & 2.789424 & 3.184789 & -1.422869 \\
\hline 8 & 1 & 0.944554 & 1.519282 & -1.517874 \\
\hline 9 & 1 & 0.447137 & 2.865831 & -0.506919 \\
\hline 10 & 1 & 1.022226 & 1.642319 & 1.541701 \\
\hline 11 & 1 & 3.518415 & 0.824755 & -1.507465 \\
\hline 12 & 1 & 4.632389 & 1.735539 & -0.496058 \\
\hline 13 & 1 & 3.490295 & 0.982776 & 1.546649 \\
\hline 14 & 1 & 1.718706 & -0.629997 & 1.606122 \\
\hline 15 & 1 & 2.773125 & 3.151090 & 0.341261 \\
\hline 16 & 6 & 4.344518 & -0.780995 & 0.669571 \\
\hline 17 & 1 & 5.373479 & -0.398429 & 0.593261 \\
\hline 18 & 1 & 4.265565 & -1.266643 & 1.653852 \\
\hline 19 & 6 & 4.084262 & -1.838303 & -0.414812 \\
\hline 20 & 1 & 4.283064 & -1.411110 & -1.408200 \\
\hline 21 & 1 & 4.786706 & -2.675103 & -0.293112 \\
\hline 22 & 6 & 1.628443 & -1.178514 & -0.457273 \\
\hline 23 & 1 & 1.701272 & -0.720461 & -1.451723 \\
\hline 24 & 1 & 0.602850 & -1.547304 & -0.374349 \\
\hline 25 & 6 & 2.632985 & -2.337160 & -0.357241 \\
\hline 26 & 1 & 2.449150 & -3.066101 & -1.158132 \\
\hline 27 & 1 & 2.475620 & -2.872784 & 0.591865 \\
\hline 28 & 8 & -1.018666 & 0.280606 & -0.942067 \\
\hline 29 & 44 & -2.378697 & -0.075265 & 0.105627 \\
\hline 30 & 8 & -3.482830 & 1.227815 & 0.288388 \\
\hline 31 & 8 & -2.493920 & -1.756330 & 0.522977 \\
\hline 32 & 8 & -1.302560 & 0.375048 & 1.570527 \\
\hline 33 & 1 & -0.329668 & 0.676293 & 1.014779 \\
\hline 34 & 8 & -3.684478 & -0.617661 & -1.405825 \\
\hline 35 & 1 & -3.572448 & -1.581364 & -1.444279 \\
\hline
\end{tabular}

Sum of electronic and thermal Enthalpies $=\quad-863.164128$ Sum of electronic and thermal Free Energies $=\quad-863.226468$ NIMAG $=1, \quad-961.0832 \mathrm{~cm}^{-1}$ 
TS7a (trans-decalin, tertiary)

\begin{tabular}{|c|c|c|c|c|}
\hline Center & Atomic & \multicolumn{3}{|c|}{ Coordinates (Angstroms) } \\
\hline Number & Number & $\mathrm{X}$ & $\mathrm{Y}$ & Z \\
\hline 1 & 6 & -1.599295 & 1.291319 & 1.536923 \\
\hline 2 & 6 & -1.608562 & 0.005927 & 0.727359 \\
\hline 3 & 6 & -2.699736 & 0.006328 & -0.360633 \\
\hline 4 & 6 & -2.664927 & 1.281824 & -1.223133 \\
\hline 5 & 6 & -2.670985 & 2.561236 & -0.373756 \\
\hline 6 & 6 & -1.530582 & 2.548190 & 0.655854 \\
\hline 7 & 1 & -0.504614 & 0.007617 & -0.185525 \\
\hline 8 & 1 & -2.540067 & 1.326934 & 2.124263 \\
\hline 9 & 1 & -0.771818 & 1.274106 & 2.250654 \\
\hline 10 & 1 & -1.754906 & 1.257813 & -1.833587 \\
\hline 11 & 1 & -3.522768 & 1.277461 & -1.912047 \\
\hline 12 & 1 & -2.590423 & 3.443732 & -1.023694 \\
\hline 13 & 1 & -3.634861 & 2.647772 & 0.154297 \\
\hline 14 & 1 & -0.564761 & 2.564887 & 0.136190 \\
\hline 15 & 1 & -1.575302 & 3.448117 & 1.285067 \\
\hline 16 & 1 & -3.660976 & 0.007958 & 0.197363 \\
\hline 17 & 6 & -2.666584 & -1.272186 & -1.218662 \\
\hline 18 & 1 & -1.754311 & -1.252701 & -1.826142 \\
\hline 19 & 1 & -3.521706 & -1.267262 & -1.910937 \\
\hline 20 & 6 & -1.611555 & -1.274573 & 1.544512 \\
\hline 21 & 1 & -0.788159 & -1.259715 & 2.262739 \\
\hline 22 & 1 & -2.556202 & -1.300566 & 2.126177 \\
\hline 23 & 6 & -1.546183 & -2.536869 & 0.671229 \\
\hline 24 & 1 & -1.600614 & -3.432683 & 1.305350 \\
\hline 25 & 1 & -0.576587 & -2.563658 & 0.159042 \\
\hline 26 & 6 & -2.680487 & -2.548843 & -0.365168 \\
\hline 27 & 1 & -2.599394 & -3.433676 & -1.011798 \\
\hline 28 & 1 & -3.647549 & -2.630464 & 0.157768 \\
\hline 29 & 8 & 0.238036 & 0.016407 & -1.068966 \\
\hline 30 & 8 & 2.354449 & 1.574251 & -0.705027 \\
\hline 31 & 8 & 2.335530 & -1.591965 & -0.725312 \\
\hline 32 & 8 & 1.042523 & -0.020614 & 1.344811 \\
\hline 33 & 44 & 1.898981 & -0.018600 & -0.184885 \\
\hline 34 & 8 & 3.810063 & -0.048808 & 0.589983 \\
\hline 35 & 1 & 4.038429 & 0.894443 & 0.628720 \\
\hline
\end{tabular}

Sum of electronic and thermal Enthalpies $=\quad-863.174695$ Sum of electronic and thermal Free Energies = -863.236865 NIMAG $=1, \quad-874.6552 \mathrm{~cm}^{-1}$ 
TS7b (trans-decalin, secondary)

\begin{tabular}{|c|c|c|c|c|}
\hline Center & Atomic & \multicolumn{3}{|c|}{ Coordinates (Angstroms) } \\
\hline Number & Number & $\mathrm{X}$ & $\mathrm{Y}$ & Z \\
\hline 1 & 6 & -1.999373 & -1.619680 & -0.339408 \\
\hline 2 & 6 & -2.051835 & -0.158729 & 0.136322 \\
\hline 3 & 6 & -3.404354 & 0.506519 & -0.250627 \\
\hline 4 & 6 & -4.588599 & -0.322663 & 0.278185 \\
\hline 5 & 6 & -4.530296 & -1.784155 & -0.192045 \\
\hline 6 & 6 & -3.190251 & -2.437748 & 0.180528 \\
\hline 7 & 1 & -2.000155 & -0.160470 & 1.237950 \\
\hline 8 & 1 & -1.998860 & -1.636958 & -1.440690 \\
\hline 9 & 1 & -1.051048 & -2.060955 & -0.014981 \\
\hline 10 & 1 & -4.577198 & -0.296484 & 1.379024 \\
\hline 11 & 1 & -5.536541 & 0.140908 & -0.033565 \\
\hline 12 & 1 & -5.369034 & -2.352964 & 0.233719 \\
\hline 13 & 1 & -4.655396 & -1.815462 & -1.285336 \\
\hline 14 & 1 & -3.121884 & -2.519278 & 1.275762 \\
\hline 15 & 1 & -3.147452 & -3.463561 & -0.210160 \\
\hline 16 & 1 & -3.462634 & 0.511391 & -1.352750 \\
\hline 17 & 6 & -3.451064 & 1.966602 & 0.228934 \\
\hline 18 & 1 & -3.437067 & 1.979771 & 1.330178 \\
\hline 19 & 1 & -4.402585 & 2.428482 & -0.074911 \\
\hline 20 & 6 & -0.890481 & 0.678216 & -0.397024 \\
\hline 21 & 1 & 0.104572 & 0.030732 & 0.322849 \\
\hline 22 & 1 & -0.774176 & 0.568744 & -1.474543 \\
\hline 23 & 6 & -0.915153 & 2.122938 & 0.055911 \\
\hline 24 & 1 & -0.082238 & 2.669323 & -0.396027 \\
\hline 25 & 1 & -0.774830 & 2.169250 & 1.146176 \\
\hline 26 & 6 & -2.265112 & 2.785746 & -0.301575 \\
\hline 27 & 1 & -2.301142 & 3.809809 & 0.097029 \\
\hline 28 & 1 & -2.345900 & 2.871352 & -1.395415 \\
\hline 29 & 8 & 0.851312 & -0.523873 & 1.015728 \\
\hline 30 & 8 & 1.515935 & 0.695416 & -1.104675 \\
\hline 31 & 8 & 2.760412 & -1.824882 & -0.299614 \\
\hline 32 & 8 & 3.120922 & 0.851790 & 1.314549 \\
\hline 33 & 44 & 2.452892 & -0.166707 & 0.103468 \\
\hline 34 & 8 & 4.291219 & 0.139953 & -0.797140 \\
\hline 35 & 1 & 4.407011 & -0.667997 & -1.322908 \\
\hline
\end{tabular}

Sum of electronic and thermal Enthalpies $=\quad-863.173600$ Sum of electronic and thermal Free Energies $=-863.236405$ NIMAG $=1, \quad-871.9481 \mathrm{~cm}^{-1}$ 
C) Intermediates

Oxidation with $\mathrm{RuO}_{4}$

IMla (adamantane, tertiary)

\begin{tabular}{|c|c|c|c|c|}
\hline Center & Atomic & \multicolumn{3}{|c|}{ Coordinates (Angstroms) } \\
\hline Number & Number & $\mathrm{X}$ & $\mathrm{Y}$ & Z \\
\hline 1 & 6 & 2.769941 & -1.678091 & 0.183638 \\
\hline 2 & 1 & 3.817049 & -2.008668 & 0.194800 \\
\hline 3 & 1 & 2.150395 & -2.576909 & 0.306863 \\
\hline 4 & 6 & 2.448518 & -0.991668 & -1.160572 \\
\hline 5 & 1 & 2.621699 & -1.692783 & -1.986514 \\
\hline 6 & 6 & 2.514941 & -0.692834 & 1.343618 \\
\hline 7 & 1 & 2.736810 & -1.180647 & 2.301018 \\
\hline 8 & 6 & 3.411731 & 0.550968 & 1.169548 \\
\hline 9 & 1 & 4.469673 & 0.257634 & 1.195809 \\
\hline 10 & 1 & 3.252988 & 1.251592 & 2.000459 \\
\hline 11 & 6 & 3.345962 & 0.251296 & -1.332571 \\
\hline 12 & 1 & 3.140271 & 0.737281 & -2.295981 \\
\hline 13 & 1 & 4.402837 & -0.046816 & -1.343992 \\
\hline 14 & 6 & 3.087358 & 1.235499 & -0.173751 \\
\hline 15 & 1 & 3.713204 & 2.128578 & -0.296860 \\
\hline 16 & 6 & 1.602704 & 1.662645 & -0.186883 \\
\hline 17 & 1 & 1.395314 & 2.371757 & 0.624047 \\
\hline 18 & 1 & 1.348723 & 2.158524 & -1.131920 \\
\hline 19 & 6 & 0.963612 & -0.560943 & -1.172686 \\
\hline 20 & 1 & 0.320495 & -1.445061 & -1.073608 \\
\hline 21 & 1 & 0.705367 & -0.075802 & -2.122366 \\
\hline 22 & 6 & 1.030512 & -0.260225 & 1.332658 \\
\hline 23 & 1 & 0.381376 & -1.132648 & 1.481781 \\
\hline 24 & 1 & 0.823583 & 0.441356 & 2.150787 \\
\hline 25 & 6 & 0.707264 & 0.416359 & -0.011452 \\
\hline 26 & 1 & -2.340630 & 2.202930 & 0.007539 \\
\hline 27 & 8 & -3.059899 & 1.535627 & 0.020733 \\
\hline 28 & 44 & -2.194575 & -0.142570 & 0.000569 \\
\hline 29 & 8 & -2.348462 & -0.912200 & 1.498175 \\
\hline 30 & 8 & -2.411474 & -0.905534 & -1.491960 \\
\hline 31 & 8 & -0.639304 & 0.942700 & -0.025222 \\
\hline
\end{tabular}

Sum of electronic and thermal Enthalpies $=\quad-786.231715$ Sum of electronic and thermal Free Energies $=\quad-786.286795$ 
IM1b (adamantane, secondary)

\begin{tabular}{|c|c|c|c|c|}
\hline Center & Atomic & \multicolumn{3}{|c|}{ Coordinates (Angstroms) } \\
\hline Number & Number & $\mathrm{X}$ & $\mathrm{Y}$ & $\mathrm{Z}$ \\
\hline 1 & 6 & -0.584253 & -0.554861 & 0.312046 \\
\hline 2 & 1 & -0.303139 & -1.620706 & 0.344369 \\
\hline 3 & 1 & 1.070715 & -1.713475 & -1.027705 \\
\hline 4 & 6 & -1.658832 & -0.321060 & 1.401155 \\
\hline 5 & 1 & -1.219847 & -0.564017 & 2.376144 \\
\hline 6 & 6 & -1.165509 & -0.192175 & -1.074945 \\
\hline 7 & 1 & -0.393694 & -0.333791 & -1.844387 \\
\hline 8 & 6 & -2.362647 & -1.127077 & -1.357592 \\
\hline 9 & 1 & -2.036863 & -2.177308 & -1.372050 \\
\hline 10 & 1 & -2.767786 & -0.908389 & -2.354179 \\
\hline 11 & 6 & -2.852112 & -1.256537 & 1.103567 \\
\hline 12 & 1 & -3.611965 & -1.131424 & 1.886183 \\
\hline 13 & 1 & -2.532854 & -2.307699 & 1.137085 \\
\hline 14 & 6 & -3.447412 & -0.922146 & -0.279934 \\
\hline 15 & 1 & -4.296707 & -1.586609 & -0.484330 \\
\hline 16 & 6 & -3.916925 & 0.548256 & -0.296915 \\
\hline 17 & 1 & -4.357358 & 0.790539 & -1.273873 \\
\hline 18 & 1 & -4.704202 & 0.698947 & 0.454374 \\
\hline 19 & 6 & -2.128970 & 1.147898 & 1.377829 \\
\hline 20 & 1 & -1.289956 & 1.815078 & 1.605183 \\
\hline 21 & 1 & -2.883421 & 1.297949 & 2.161735 \\
\hline 22 & 6 & -1.635150 & 1.277691 & -1.082667 \\
\hline 23 & 1 & -0.785822 & 1.946274 & -0.898981 \\
\hline 24 & 1 & -2.029997 & 1.524842 & -2.077105 \\
\hline 25 & 6 & -2.721955 & 1.481912 & -0.006879 \\
\hline 26 & 1 & -3.058204 & 2.526498 & -0.020319 \\
\hline 27 & 8 & 2.005710 & -1.445276 & -1.068482 \\
\hline 28 & 8 & 0.547194 & 0.251159 & 0.664669 \\
\hline 29 & 8 & 3.272123 & -0.430625 & 1.335487 \\
\hline 30 & 8 & 2.636702 & 1.391425 & -0.980381 \\
\hline 31 & 44 & 2.326133 & 0.070873 & 0.027353 \\
\hline
\end{tabular}

Sum of electronic and thermal Enthalpies $=\quad-786.217137$ Sum of electronic and thermal Free Energies $=\quad-786.272339$ 
IM2a (cis-decalin, tertiary)

\begin{tabular}{|c|c|c|c|c|}
\hline Center & Atomic & \multicolumn{3}{|c|}{ Coordinates (Angstroms) } \\
\hline Number & Number & $\mathrm{X}$ & $\mathrm{Y}$ & Z \\
\hline 1 & 6 & 1.032795 & -1.721389 & 0.329534 \\
\hline 2 & 6 & 2.430246 & -2.234123 & 0.711666 \\
\hline 3 & 6 & 3.475571 & -1.809073 & -0.325955 \\
\hline 4 & 6 & 3.457202 & -0.289220 & -0.527677 \\
\hline 5 & 6 & 2.061540 & 0.236058 & -0.929841 \\
\hline 6 & 6 & 0.961263 & -0.196121 & 0.095761 \\
\hline 7 & 1 & 4.475036 & -2.140251 & -0.018003 \\
\hline 8 & 1 & 2.715916 & -1.852162 & 1.701531 \\
\hline 9 & 1 & 2.397364 & -3.326718 & 0.801464 \\
\hline 10 & 1 & 0.712401 & -2.205633 & -0.602205 \\
\hline 11 & 1 & 0.309003 & -2.028318 & 1.096278 \\
\hline 12 & 1 & 3.804021 & 0.202972 & 0.390455 \\
\hline 13 & 1 & 4.167929 & -0.002728 & -1.313679 \\
\hline 14 & 1 & 1.792613 & -0.252706 & -1.876411 \\
\hline 15 & 1 & 3.259699 & -2.306350 & -1.282617 \\
\hline 16 & 6 & 2.047206 & 1.761803 & -1.160252 \\
\hline 17 & 1 & 2.871407 & 2.027531 & -1.835173 \\
\hline 18 & 1 & 1.118103 & 2.030118 & -1.676796 \\
\hline 19 & 6 & 2.144911 & 2.570394 & 0.142434 \\
\hline 20 & 1 & 3.131059 & 2.427116 & 0.606161 \\
\hline 21 & 1 & 2.063404 & 3.641376 & -0.079914 \\
\hline 22 & 6 & 1.068807 & 0.639123 & 1.389901 \\
\hline 23 & 1 & 1.999158 & 0.367284 & 1.904230 \\
\hline 24 & 1 & 0.256870 & 0.374049 & 2.079549 \\
\hline 25 & 6 & 1.048716 & 2.155089 & 1.133195 \\
\hline 26 & 1 & 1.171631 & 2.683617 & 2.086501 \\
\hline 27 & 1 & 0.066942 & 2.443532 & 0.737556 \\
\hline 28 & 8 & -0.261885 & 0.124755 & -0.613966 \\
\hline 29 & 44 & -2.097773 & -0.082439 & -0.177064 \\
\hline 30 & 8 & -2.698617 & -1.288721 & -1.198915 \\
\hline 31 & 8 & -1.994288 & -0.840417 & 1.561821 \\
\hline 32 & 8 & -2.708789 & 1.484534 & 0.010887 \\
\hline 33 & 1 & -1.071450 & -0.971851 & 1.840656 \\
\hline
\end{tabular}

Sum of electronic and thermal Enthalpies $=\quad-787.396781$

Sum of electronic and thermal Free Energies $=\quad-787.455047$ 
IM2b (cis-decalin, secondary)

\begin{tabular}{|c|c|c|c|c|}
\hline \multirow{2}{*}{$\begin{array}{l}\text { Center } \\
\text { Number }\end{array}$} & \multirow{2}{*}{$\begin{array}{l}\text { Atomic } \\
\text { Number }\end{array}$} & \multicolumn{3}{|c|}{ Coordinates (Angstroms) } \\
\hline & & $\mathrm{X}$ & $\mathrm{Y}$ & Z \\
\hline 1 & 6 & -0.367937 & 0.434614 & -0.221724 \\
\hline 2 & 6 & -0.528610 & 1.581818 & 0.784712 \\
\hline 3 & 6 & -1.686234 & 2.505500 & 0.370544 \\
\hline 4 & 6 & -2.994046 & 1.722605 & 0.180948 \\
\hline 5 & 6 & -2.825205 & 0.555414 & -0.812742 \\
\hline 6 & 6 & -1.664674 & -0.391971 & -0.398367 \\
\hline 7 & 1 & -1.816347 & 3.294421 & 1.120825 \\
\hline 8 & 1 & -0.712591 & 1.158028 & 1.779173 \\
\hline 9 & 1 & 0.407683 & 2.150091 & 0.861286 \\
\hline 10 & 1 & -0.133707 & 0.850838 & -1.216769 \\
\hline 11 & 1 & -3.334882 & 1.350847 & 1.155527 \\
\hline 12 & 1 & -3.781494 & 2.395076 & -0.183410 \\
\hline 13 & 1 & -2.554735 & 1.002361 & -1.782659 \\
\hline 14 & 1 & -1.480621 & -1.080822 & -1.235947 \\
\hline 15 & 1 & -1.427359 & 3.013531 & -0.570742 \\
\hline 16 & 6 & -4.123484 & -0.253201 & -1.022930 \\
\hline 17 & 1 & -4.948760 & 0.433374 & -1.254493 \\
\hline 18 & 1 & -3.995475 & -0.893744 & -1.907394 \\
\hline 19 & 6 & -4.486319 & -1.141158 & 0.178312 \\
\hline 20 & 1 & -4.757469 & -0.516002 & 1.040392 \\
\hline 21 & 1 & -5.376436 & -1.737129 & -0.059647 \\
\hline 22 & 6 & -2.030482 & -1.259298 & 0.823064 \\
\hline 23 & 1 & -2.171843 & -0.634677 & 1.714804 \\
\hline 24 & 1 & -1.200002 & -1.936235 & 1.047462 \\
\hline 25 & 6 & -3.317009 & -2.059429 & 0.562190 \\
\hline 26 & 1 & -3.572788 & -2.653761 & 1.448290 \\
\hline 27 & 1 & -3.136520 & -2.776598 & -0.252393 \\
\hline 28 & 8 & 0.661922 & -0.481138 & 0.173490 \\
\hline 29 & 44 & 2.537917 & -0.261127 & -0.017997 \\
\hline 30 & 8 & 3.185907 & -0.054239 & 1.528693 \\
\hline 31 & 8 & 3.051082 & -1.384875 & -1.171676 \\
\hline 32 & 8 & 2.466488 & 1.440308 & -0.857745 \\
\hline 33 & 1 & 1.545834 & 1.740160 & -0.958592 \\
\hline
\end{tabular}

Sum of electronic and thermal Enthalpies $=\quad-787.396370$ Sum of electronic and thermal Free Energies $=\quad-787.455640$ 
IM3a (trans-decalin, tertiary)

\begin{tabular}{|c|c|c|c|c|}
\hline $\begin{array}{l}\text { Center } \\
\text { Number }\end{array}$ & $\begin{array}{l}\text { Atomic } \\
\text { Number }\end{array}$ & \multicolumn{3}{|c|}{ Coordinates (Angstroms) } \\
\hline 1 & 6 & -0.938484 & -1.277215 & -1.434757 \\
\hline 2 & 6 & -1.034224 & 0.000030 & -0.579508 \\
\hline 3 & 6 & -2.369993 & 0.000451 & 0.226994 \\
\hline 4 & 6 & -2.535234 & -1.284521 & 1.055248 \\
\hline 5 & 6 & -2.427992 & -2.544528 & 0.183101 \\
\hline 6 & 6 & -1.116741 & -2.564644 & -0.615737 \\
\hline 7 & 1 & 1.062305 & -0.000701 & 2.283963 \\
\hline 8 & 1 & -1.723449 & -1.216654 & -2.201048 \\
\hline 9 & 1 & 0.016878 & -1.293759 & -1.974832 \\
\hline 10 & 1 & -1.768524 & -1.312599 & 1.840741 \\
\hline 11 & 1 & -3.506922 & -1.258234 & 1.565661 \\
\hline 12 & 1 & -2.506557 & -3.444344 & 0.805668 \\
\hline 13 & 1 & -3.278101 & -2.572360 & -0.515056 \\
\hline 14 & 1 & -0.267318 & -2.682574 & 0.068993 \\
\hline 15 & 1 & -1.093173 & -3.429232 & -1.290319 \\
\hline 16 & 1 & -3.158489 & 0.000684 & -0.543517 \\
\hline 17 & 6 & -2.534430 & 1.285558 & 1.055199 \\
\hline 18 & 1 & -1.767733 & 1.313166 & 1.840718 \\
\hline 19 & 1 & -3.506153 & 1.259904 & 1.565579 \\
\hline 20 & 6 & -0.937923 & 1.277114 & -1.434935 \\
\hline 21 & 1 & 0.017279 & 1.293034 & -1.975314 \\
\hline 22 & 1 & -1.723170 & 1.216917 & -2.200965 \\
\hline 23 & 6 & -1.115226 & 2.564718 & -0.615990 \\
\hline 24 & 1 & -1.091210 & 3.429240 & -1.290642 \\
\hline 25 & 1 & -0.265634 & 2.682164 & 0.068603 \\
\hline 26 & 6 & -2.426380 & 2.545496 & 0.183036 \\
\hline 27 & 1 & -2.504189 & 3.445352 & 0.805644 \\
\hline 28 & 1 & -3.276578 & 2.573980 & -0.514983 \\
\hline 29 & 8 & 1.986935 & -0.000662 & 1.956402 \\
\hline 30 & 44 & 1.858595 & -0.000229 & 0.072257 \\
\hline 31 & 8 & 2.329637 & -1.498386 & -0.554203 \\
\hline 32 & 8 & 2.329927 & 1.498145 & -0.553468 \\
\hline 33 & 8 & 0.001290 & -0.000180 & 0.444507 \\
\hline
\end{tabular}

Sum of electronic and thermal Enthalpies $=\quad-787.412504$

Sum of electronic and thermal Free Energies $=\quad-\quad-787.470633$ 
IM3b (trans-decalin, secondary)

\begin{tabular}{|c|c|c|c|c|}
\hline \multirow{2}{*}{$\begin{array}{l}\text { Center } \\
\text { Number }\end{array}$} & \multirow{2}{*}{$\begin{array}{l}\text { Atomic } \\
\text { Number }\end{array}$} & \multicolumn{3}{|c|}{ Coordinates (Angstroms) } \\
\hline & & $\mathrm{X}$ & $\mathrm{Y}$ & $\mathrm{Z}$ \\
\hline 1 & 6 & 1.474599 & -1.503535 & 0.836219 \\
\hline 2 & 6 & 1.351371 & -0.113952 & 0.183746 \\
\hline 3 & 6 & 2.742985 & 0.559213 & 0.051606 \\
\hline 4 & 6 & 3.709271 & -0.354446 & -0.723081 \\
\hline 5 & 6 & 3.827682 & -1.747267 & -0.088095 \\
\hline 6 & 6 & 2.450114 & -2.407137 & 0.067452 \\
\hline 7 & 1 & 0.955162 & -0.246766 & -0.836636 \\
\hline 8 & 1 & 1.829248 & -1.378699 & 1.871296 \\
\hline 9 & 1 & 0.490858 & -1.981090 & 0.895106 \\
\hline 10 & 1 & 3.350976 & -0.454484 & -1.759084 \\
\hline 11 & 1 & 4.696348 & 0.123409 & -0.780794 \\
\hline 12 & 1 & 4.489293 & -2.381978 & -0.690984 \\
\hline 13 & 1 & 4.299752 & -1.655331 & 0.901330 \\
\hline 14 & 1 & 2.033030 & -2.620383 & -0.927636 \\
\hline 15 & 1 & 2.544703 & -3.373531 & 0.578170 \\
\hline 16 & 1 & 3.145547 & 0.679237 & 1.072233 \\
\hline 17 & 6 & 2.637355 & 1.958678 & -0.574173 \\
\hline 18 & 1 & 2.287197 & 1.864956 & -1.613418 \\
\hline 19 & 1 & 3.633253 & 2.418124 & -0.621467 \\
\hline 20 & 6 & 0.387693 & 0.813029 & 0.945471 \\
\hline 21 & 1 & -2.713397 & -0.538012 & 1.967644 \\
\hline 22 & 1 & 0.753415 & 0.910520 & 1.979481 \\
\hline 23 & 6 & 0.286322 & 2.211691 & 0.328901 \\
\hline 24 & 1 & -0.385389 & 2.827116 & 0.938926 \\
\hline 25 & 1 & -0.165353 & 2.139196 & -0.669761 \\
\hline 26 & 6 & 1.674039 & 2.860575 & 0.208476 \\
\hline 27 & 1 & 1.581159 & 3.839620 & -0.276428 \\
\hline 28 & 1 & 2.081374 & 3.047032 & 1.212696 \\
\hline 29 & 8 & -3.214637 & -0.733577 & 1.146976 \\
\hline 30 & 44 & -2.083699 & -0.218638 & -0.274158 \\
\hline 31 & 8 & -0.917423 & 0.227607 & 1.152583 \\
\hline 32 & 8 & -2.671804 & 1.175904 & -1.026717 \\
\hline 33 & 8 & -1.505261 & -1.577429 & -1.097452 \\
\hline
\end{tabular}

Sum of electronic and thermal Enthalpies $=\quad-787.411057$

Sum of electronic and thermal Free Energies $=\quad-\quad 787.470070$ 
IM4 (methane)

\begin{tabular}{|c|c|c|c|c|}
\hline Center & Atomic & \multicolumn{3}{|c|}{ Coordinates (Angstroms) } \\
\hline Number & Number & $\mathrm{X}$ & Y & $\mathrm{Z}$ \\
\hline 1 & 6 & 2.521561 & 0.000099 & -0.174854 \\
\hline 2 & 1 & 2.656144 & 0.902811 & 0.437431 \\
\hline 3 & 1 & 1.202122 & -0.001376 & 1.751921 \\
\hline 4 & 1 & 3.295752 & 0.000263 & -0.949774 \\
\hline 5 & 1 & 2.656184 & -0.902864 & 0.437055 \\
\hline 6 & 44 & -0.429756 & 0.000044 & -0.046248 \\
\hline 7 & 8 & 0.228522 & -0.001056 & 1.733028 \\
\hline 8 & 8 & 1.281579 & 0.000259 & -0.869909 \\
\hline 9 & 8 & -1.132164 & -1.506011 & -0.344456 \\
\hline 10 & 8 & -1.131727 & 1.506641 & -0.342737 \\
\hline Sum of & lectronic and & al & pies $=$ & -436.204170 \\
\hline Sum of & electronic and & thermal Free & Energies $=$ & -436.245473 \\
\hline
\end{tabular}

Oxidation with $\mathrm{RuO}_{4}(\mathrm{OH})$

IM8 (methane)

\begin{tabular}{ccrrr}
$\begin{array}{c}\text { Center } \\
\text { Number }\end{array}$ & $\begin{array}{c}\text { Atomic } \\
\text { Number }\end{array}$ & \multicolumn{2}{c}{ Coordinates } & \multicolumn{1}{c}{ (Angstroms) } \\
\hline 1 & 6 & 2.628970 & -0.148597 & -0.149440 \\
2 & 1 & 2.712296 & 0.564206 & -0.981618 \\
3 & 1 & -0.181881 & 2.107496 & -0.736672 \\
4 & 1 & 3.455986 & -0.874239 & -0.228326 \\
5 & 1 & 2.760139 & 0.418936 & 0.786449 \\
6 & 4 & -0.296728 & 0.009858 & 0.127084 \\
7 & 8 & 0.601686 & 1.659811 & -0.346047 \\
8 & 8 & 1.429790 & -0.879505 & -0.185788 \\
9 & 8 & -0.673062 & 0.043176 & 1.770091 \\
10 & 8 & -1.592435 & 0.743495 & -0.919619 \\
11 & 8 & -0.974403 & -1.629203 & -0.632626 \\
12 & 1 & -1.796926 & -1.260779 & -1.022967 \\
\hline
\end{tabular}

Sum of electronic and thermal Enthalpies =

$-512.089572$

Sum of electronic and thermal Free Energies =

$-512.133619$ 
IM5a (adamantane, tertiary)

\begin{tabular}{|c|c|c|c|c|}
\hline $\begin{array}{l}\text { Center } \\
\text { Number }\end{array}$ & $\begin{array}{l}\text { Atomic } \\
\text { Number }\end{array}$ & \multicolumn{3}{|c|}{ Coordinates (Angstroms) } \\
\hline 1 & 6 & 2.935856 & -1.655734 & 0.029889 \\
\hline 2 & 1 & 3.983493 & -1.995125 & 0.042421 \\
\hline 3 & 1 & 2.301707 & -2.551598 & 0.041864 \\
\hline 4 & 6 & 2.658360 & -0.834293 & -1.247731 \\
\hline 5 & 1 & 2.871170 & -1.451016 & -2.133163 \\
\hline 6 & 6 & 2.643130 & -0.790390 & 1.274795 \\
\hline 7 & 1 & 2.844314 & -1.376027 & 2.183589 \\
\hline 8 & 6 & 3.550256 & 0.456757 & 1.254838 \\
\hline 9 & 1 & 4.610360 & 0.160868 & 1.281761 \\
\hline 10 & 1 & 3.365228 & 1.069870 & 2.148618 \\
\hline 11 & 6 & 3.565216 & 0.413149 & -1.260109 \\
\hline 12 & 1 & 3.390446 & 0.995157 & -2.176481 \\
\hline 13 & 1 & 4.625810 & 0.117597 & -1.264427 \\
\hline 14 & 6 & 3.263476 & 1.279805 & -0.018846 \\
\hline 15 & 1 & 3.899941 & 2.177221 & -0.030631 \\
\hline 16 & 6 & 1.777831 & 1.702305 & -0.034284 \\
\hline 17 & 1 & 1.544889 & 2.327717 & 0.838080 \\
\hline 18 & 1 & 1.554659 & 2.297891 & -0.929653 \\
\hline 19 & 6 & 1.177177 & -0.400103 & -1.265567 \\
\hline 20 & 1 & 0.507774 & -1.265189 & -1.261155 \\
\hline 21 & 1 & 0.961736 & 0.194849 & -2.164668 \\
\hline 22 & 6 & 1.161104 & -0.356207 & 1.260151 \\
\hline 23 & 1 & 0.498277 & -1.225353 & 1.289982 \\
\hline 24 & 1 & 0.938260 & 0.270872 & 2.135368 \\
\hline 25 & 6 & 0.860042 & 0.454980 & -0.019054 \\
\hline 26 & 1 & -2.090124 & 2.328163 & -0.154787 \\
\hline 27 & 8 & -2.898464 & 1.785271 & -0.167498 \\
\hline 28 & 44 & -2.137057 & -0.039778 & 0.028848 \\
\hline 29 & 8 & -2.767677 & -0.293399 & 1.587977 \\
\hline 30 & 8 & -2.839095 & -0.524230 & -1.472466 \\
\hline 31 & 8 & -0.465807 & 0.962967 & -0.040805 \\
\hline 32 & 8 & -1.212731 & -1.867878 & 0.023576 \\
\hline 33 & 1 & -1.861921 & -2.449125 & -0.406775 \\
\hline
\end{tabular}

Sum of electronic and thermal Enthalpies =

$-862.102579$

Sum of electronic and thermal Free Energies =

$-862.160716$ 
IM5b (adamantane, secondary)

\begin{tabular}{|c|c|c|c|c|}
\hline \multirow{2}{*}{$\begin{array}{l}\text { Center } \\
\text { Number }\end{array}$} & \multirow{2}{*}{$\begin{array}{l}\text { Atomic } \\
\text { Number }\end{array}$} & \multicolumn{3}{|c|}{ Coordinates (Angstroms) } \\
\hline & & $\mathrm{X}$ & $\mathrm{Y}$ & Z \\
\hline 1 & 6 & -0.747858 & 0.280882 & -0.548213 \\
\hline 2 & 1 & -0.442036 & 1.194569 & -1.075329 \\
\hline 3 & 1 & 2.502051 & 2.087864 & -0.728177 \\
\hline 4 & 6 & -1.854031 & -0.425712 & -1.373783 \\
\hline 5 & 1 & -1.422859 & -0.718933 & -2.339511 \\
\hline 6 & 6 & -1.336582 & 0.685270 & 0.828094 \\
\hline 7 & 1 & -0.546450 & 1.175267 & 1.408754 \\
\hline 8 & 6 & -2.511411 & 1.661370 & 0.609069 \\
\hline 9 & 1 & -2.158363 & 2.565439 & 0.093243 \\
\hline 10 & 1 & -2.921753 & 1.985420 & 1.577795 \\
\hline 11 & 6 & -3.027308 & 0.553318 & -1.589502 \\
\hline 12 & 1 & -3.808056 & 0.079306 & -2.204357 \\
\hline 13 & 1 & -2.682918 & 1.441597 & -2.138509 \\
\hline 14 & 6 & -3.612886 & 0.974160 & -0.225110 \\
\hline 15 & 1 & -4.451507 & 1.669713 & -0.379936 \\
\hline 16 & 6 & -4.113259 & -0.280681 & 0.523188 \\
\hline 17 & 1 & -4.549589 & 0.009228 & 1.491218 \\
\hline 18 & 1 & -4.912781 & -0.769178 & -0.054840 \\
\hline 19 & 6 & -2.354854 & -1.677040 & -0.624473 \\
\hline 20 & 1 & -1.518569 & -2.369867 & -0.482670 \\
\hline 21 & 1 & -3.121615 & -2.195076 & -1.221944 \\
\hline 22 & 6 & -1.837923 & -0.569223 & 1.572040 \\
\hline 23 & 1 & -0.999958 & -1.256759 & 1.731987 \\
\hline 24 & 1 & -2.230298 & -0.289995 & 2.562203 \\
\hline 25 & 6 & -2.940292 & -1.260712 & 0.741732 \\
\hline 26 & 1 & -3.303356 & -2.151526 & 1.275592 \\
\hline 27 & 8 & 1.614148 & 1.779047 & -0.439808 \\
\hline 28 & 8 & 0.345304 & -0.597949 & -0.431801 \\
\hline 29 & 8 & 3.669033 & 0.489266 & -0.751667 \\
\hline 30 & 8 & 2.318319 & -0.013760 & 1.791011 \\
\hline 31 & 44 & 2.148121 & 0.001820 & 0.114968 \\
\hline 32 & 8 & 2.619635 & -1.738547 & -0.568258 \\
\hline 33 & 1 & 3.537663 & -1.531379 & -0.848162 \\
\hline
\end{tabular}

Sum of electronic and thermal Enthalpies $=\quad-862.104452$ Sum of electronic and thermal Free Energies $=\quad-862.162360$ 
IM6a (cis-decalin, tertiary)

\begin{tabular}{|c|c|c|c|c|}
\hline \multirow{2}{*}{$\begin{array}{l}\text { Center } \\
\text { Number }\end{array}$} & Atomic & \multicolumn{3}{|c|}{ Coordinates (Angstroms) } \\
\hline & Number & $\mathrm{X}$ & $\mathrm{Y}$ & Z \\
\hline 1 & 6 & -1.280623 & -1.776049 & -0.209808 \\
\hline 2 & 6 & -2.692726 & -2.242883 & -0.594294 \\
\hline 3 & 6 & -3.740026 & -1.719781 & 0.397780 \\
\hline 4 & 6 & -3.656986 & -0.192361 & 0.521969 \\
\hline 5 & 6 & -2.244785 & 0.286523 & 0.920167 \\
\hline 6 & 6 & -1.127417 & -0.243408 & -0.043804 \\
\hline 7 & 1 & -4.752590 & -2.025342 & 0.095086 \\
\hline 8 & 1 & -2.947594 & -1.894681 & -1.606122 \\
\hline 9 & 1 & -2.721819 & -3.341427 & -0.633236 \\
\hline 10 & 1 & -1.010229 & -2.219723 & 0.758990 \\
\hline 11 & 1 & -0.537074 & -2.118028 & -0.934125 \\
\hline 12 & 1 & -3.962047 & 0.258851 & -0.432318 \\
\hline 13 & 1 & -4.378077 & 0.166483 & 1.272111 \\
\hline 14 & 1 & -2.015746 & -0.154850 & 1.900533 \\
\hline 15 & 1 & -3.556670 & -2.172015 & 1.384504 \\
\hline 16 & 6 & -2.163012 & 1.819185 & 1.068452 \\
\hline 17 & 1 & -2.972066 & 2.170792 & 1.727371 \\
\hline 18 & 1 & -1.214817 & 2.058739 & 1.562657 \\
\hline 19 & 6 & -2.217611 & 2.555121 & -0.280061 \\
\hline 20 & 1 & -3.208606 & 2.424610 & -0.742191 \\
\hline 21 & 1 & -2.095612 & 3.635989 & -0.119205 \\
\hline 22 & 6 & -1.202176 & 0.504640 & -1.398187 \\
\hline 23 & 1 & -2.138575 & 0.241963 & -1.913349 \\
\hline 24 & 1 & -0.381083 & 0.152654 & -2.028002 \\
\hline 25 & 6 & -1.133348 & 2.032019 & -1.233643 \\
\hline 26 & 1 & -1.229649 & 2.513641 & -2.217763 \\
\hline 27 & 1 & -0.144676 & 2.303303 & -0.845943 \\
\hline 28 & 8 & 0.077114 & 0.036303 & 0.655569 \\
\hline 29 & 44 & 1.962661 & 0.002809 & 0.050831 \\
\hline 30 & 8 & 3.343288 & -1.180826 & 0.023019 \\
\hline 31 & 8 & 1.443671 & -1.048729 & -1.494368 \\
\hline 32 & 8 & 2.418247 & 1.537325 & -0.475197 \\
\hline 33 & 1 & 2.292895 & -1.537817 & -1.584770 \\
\hline 34 & 8 & 2.249262 & -0.113015 & 1.952958 \\
\hline 35 & 1 & 3.116538 & -0.573243 & 1.931909 \\
\hline
\end{tabular}

Sum of electronic and thermal Enthalpies $=\quad-863.281709$ Sum of electronic and thermal Free Energies = $\quad-863.341946$ 
IM6b (cis-decalin, secondary)

\begin{tabular}{|c|c|c|c|c|}
\hline Center & Atomic & \multicolumn{3}{|c|}{ Coordinates (Angstroms) } \\
\hline Number & Number & $\mathrm{X}$ & Y & Z \\
\hline 1 & 6 & 0.537001 & 0.922370 & -0.064127 \\
\hline 2 & 6 & 1.175011 & 2.074769 & -0.863091 \\
\hline 3 & 6 & 2.447012 & 2.597743 & -0.176597 \\
\hline 4 & 6 & 3.461649 & 1.469071 & 0.065988 \\
\hline 5 & 6 & 2.833553 & 0.311768 & 0.869146 \\
\hline 6 & 6 & 1.543066 & -0.226428 & 0.196742 \\
\hline 7 & 1 & 2.904632 & 3.398290 & -0.775501 \\
\hline 8 & 1 & 1.410900 & 1.725721 & -1.877535 \\
\hline 9 & 1 & 0.433620 & 2.875955 & -0.971840 \\
\hline 10 & 1 & 0.245815 & 1.319005 & 0.919243 \\
\hline 11 & 1 & 3.831774 & 1.106977 & -0.903182 \\
\hline 12 & 1 & 4.339508 & 1.855698 & 0.605496 \\
\hline 13 & 1 & 2.542212 & 0.733503 & 1.844363 \\
\hline 14 & 1 & 1.048313 & -0.892924 & 0.915965 \\
\hline 15 & 1 & 2.176380 & 3.048715 & 0.790452 \\
\hline 16 & 6 & 3.825820 & -0.837950 & 1.146023 \\
\hline 17 & 1 & 4.762026 & -0.436388 & 1.562798 \\
\hline 18 & 1 & 3.393641 & -1.488645 & 1.920726 \\
\hline 19 & 6 & 4.122270 & -1.695927 & -0.095863 \\
\hline 20 & 1 & 4.673993 & -1.099711 & -0.837390 \\
\hline 21 & 1 & 4.781685 & -2.532320 & 0.177715 \\
\hline 22 & 6 & 1.854402 & -1.068497 & -1.055334 \\
\hline 23 & 1 & 2.295569 & -0.444301 & -1.845915 \\
\hline 24 & 1 & 0.912735 & -1.453845 & -1.457688 \\
\hline 25 & 6 & 2.822964 & -2.216400 & -0.731110 \\
\hline 26 & 1 & 3.049866 & -2.795471 & -1.637640 \\
\hline 27 & 1 & 2.335638 & -2.911703 & -0.030684 \\
\hline 28 & 8 & -0.588027 & 0.467333 & -0.779465 \\
\hline 29 & 44 & -2.297976 & -0.019359 & 0.072880 \\
\hline 30 & 8 & -3.347948 & 1.295419 & 0.149378 \\
\hline 31 & 8 & -3.026086 & -1.553301 & 0.720295 \\
\hline 32 & 8 & -1.325584 & -0.124763 & 1.748135 \\
\hline 33 & 1 & -1.868777 & -0.834894 & 2.156753 \\
\hline 34 & 8 & -2.556845 & -0.917707 & -1.615577 \\
\hline 35 & 1 & -3.139152 & -1.640837 & -1.295646 \\
\hline
\end{tabular}

Sum of electronic and thermal Enthalpies= $-863.283697$ Sum of electronic and thermal Free Energies= $-863.344917$ 
IM7a (trans-decalin, tertiary)

\begin{tabular}{|c|c|c|c|c|}
\hline Center & Atomic & \multicolumn{3}{|c|}{ Coordinates (Angstroms) } \\
\hline Number & Number & $\mathrm{X}$ & $\mathrm{Y}$ & $\mathrm{Z}$ \\
\hline 1 & 6 & -1.103066 & 1.261131 & 1.454203 \\
\hline 2 & 6 & -1.188738 & -0.006748 & 0.577671 \\
\hline 3 & 6 & -2.520632 & 0.022436 & -0.241439 \\
\hline 4 & 6 & -2.639453 & 1.309368 & -1.074208 \\
\hline 5 & 6 & -2.522196 & 2.567707 & -0.200290 \\
\hline 6 & 6 & -1.229406 & 2.550528 & 0.629381 \\
\hline 7 & 1 & 0.778616 & -0.056801 & -2.322673 \\
\hline 8 & 1 & -1.916700 & 1.221642 & 2.196365 \\
\hline 9 & 1 & -0.155908 & 1.237677 & 2.000597 \\
\hline 10 & 1 & -1.842159 & 1.315342 & -1.828028 \\
\hline 11 & 1 & -3.596631 & 1.310207 & -1.617647 \\
\hline 12 & 1 & -2.566796 & 3.470131 & -0.826835 \\
\hline 13 & 1 & -3.389254 & 2.616061 & 0.478761 \\
\hline 14 & 1 & -0.362336 & 2.632247 & -0.037503 \\
\hline 15 & 1 & -1.196182 & 3.423105 & 1.297010 \\
\hline 16 & 1 & -3.333542 & 0.030948 & 0.505634 \\
\hline 17 & 6 & -2.683065 & -1.247817 & -1.092712 \\
\hline 18 & 1 & -1.887882 & -1.268617 & -1.848367 \\
\hline 19 & 1 & -3.640782 & -1.208870 & -1.633826 \\
\hline 20 & 6 & -1.148331 & -1.288626 & 1.436831 \\
\hline 21 & 1 & -0.197041 & -1.295822 & 1.976994 \\
\hline 22 & 1 & -1.961094 & -1.234239 & 2.179143 \\
\hline 23 & 6 & -1.313498 & -2.559609 & 0.591505 \\
\hline 24 & 1 & -1.308045 & -3.444379 & 1.243933 \\
\hline 25 & 1 & -0.448527 & -2.654987 & -0.076489 \\
\hline 26 & 6 & -2.605734 & -2.522903 & -0.238840 \\
\hline 27 & 1 & -2.678716 & -3.413264 & -0.879833 \\
\hline 28 & 1 & -3.473970 & -2.554737 & 0.439699 \\
\hline 29 & 8 & 1.736698 & -0.089008 & -2.153715 \\
\hline 30 & 44 & 1.774884 & 0.013879 & -0.165811 \\
\hline 31 & 8 & 2.467928 & 1.568790 & -0.131497 \\
\hline 32 & 8 & 2.584658 & -1.505797 & -0.070625 \\
\hline 33 & 8 & -0.156115 & -0.022389 & -0.412760 \\
\hline 34 & 8 & 1.654965 & -0.052969 & 1.873741 \\
\hline 35 & 1 & 2.501722 & -0.438157 & 2.154976 \\
\hline
\end{tabular}

Sum of electronic and thermal Enthalpies $=\quad-863.285605$ Sum of electronic and thermal Free Energies $=\quad-863.347668$ 
IM7b (trans-decalin, secondary)

\begin{tabular}{|c|c|c|c|c|}
\hline \multirow{2}{*}{$\begin{array}{l}\text { Center } \\
\text { Number }\end{array}$} & \multirow{2}{*}{$\begin{array}{l}\text { Atomic } \\
\text { Number }\end{array}$} & \multicolumn{3}{|c|}{ Coordinates (Angstroms) } \\
\hline & & $\mathrm{X}$ & Y & Z \\
\hline 1 & 6 & 1.586746 & -1.542107 & 0.815916 \\
\hline 2 & 6 & 1.511226 & -0.132237 & 0.205867 \\
\hline 3 & 6 & 2.919159 & 0.502197 & 0.106839 \\
\hline 4 & 6 & 3.875655 & -0.412928 & -0.678657 \\
\hline 5 & 6 & 3.947431 & -1.825699 & -0.080181 \\
\hline 6 & 6 & 2.548551 & -2.450409 & 0.035445 \\
\hline 7 & 1 & 1.089125 & -0.210819 & -0.805080 \\
\hline 8 & 1 & 1.926885 & -1.469277 & 1.863129 \\
\hline 9 & 1 & 0.584691 & -1.981790 & 0.837217 \\
\hline 10 & 1 & 3.524855 & -0.479744 & -1.719914 \\
\hline 11 & 1 & 4.879355 & 0.037146 & -0.715459 \\
\hline 12 & 1 & 4.607246 & -2.463099 & -0.686460 \\
\hline 13 & 1 & 4.402214 & -1.769927 & 0.921378 \\
\hline 14 & 1 & 2.143011 & -2.615109 & -0.973565 \\
\hline 15 & 1 & 2.612752 & -3.439124 & 0.511341 \\
\hline 16 & 1 & 3.321040 & 0.594285 & 1.133126 \\
\hline 17 & 6 & 2.852359 & 1.916483 & -0.492294 \\
\hline 18 & 1 & 2.496939 & 1.846560 & -1.530757 \\
\hline 19 & 1 & 3.861434 & 2.355060 & -0.530221 \\
\hline 20 & 6 & 0.555774 & 0.787966 & 0.995024 \\
\hline 21 & 1 & -2.460155 & -0.589913 & 2.134822 \\
\hline 22 & 1 & 0.959064 & 0.869807 & 2.023582 \\
\hline 23 & 6 & 0.500552 & 2.204262 & 0.403157 \\
\hline 24 & 1 & -0.152366 & 2.823391 & 1.032652 \\
\hline 25 & 1 & 0.044219 & 2.141325 & -0.590153 \\
\hline 26 & 6 & 1.902469 & 2.823806 & 0.303232 \\
\hline 27 & 1 & 1.845515 & 3.816946 & -0.163899 \\
\hline 28 & 1 & 2.316402 & 2.979018 & 1.312719 \\
\hline 29 & 8 & -3.068262 & -0.829681 & 1.413049 \\
\hline 30 & 44 & -2.071592 & -0.154414 & -0.169706 \\
\hline 31 & 8 & -0.734079 & 0.226588 & 1.193142 \\
\hline 32 & 8 & -3.168143 & 1.102141 & -0.503389 \\
\hline 33 & 8 & -2.040527 & -1.736967 & -0.861446 \\
\hline 34 & 8 & -0.850783 & 0.397122 & -1.718158 \\
\hline 35 & 1 & -1.157346 & -0.152148 & -2.459049 \\
\hline
\end{tabular}

Sum of electronic and thermal Enthalpies $=\quad-863.283955$ Sum of electronic and thermal Free Energies $=\quad-863.346252$ 
2) Tables for $\Delta \mathbf{E}$ values

a) Gas-phase: $\mathrm{RuO}_{4}$ (left column: transition state, right column: intermediate)

\begin{tabular}{|l|l|l|}
\hline & $\Delta E_{\text {calc }}^{\text {gas }}$ & $\Delta E_{\text {calc }}^{I M{ }_{\text {cas }}^{\text {gas }}}$ \\
\hline TS/IM1a(AD-O) & 17.0 & -47.5 \\
\hline TS/IM1b (AD- $\square)$ & 18.7 & -38.4 \\
\hline TS/IM2a (CD-O) & 16.8 & -37.8 \\
\hline TS/IM2b (CD- $\square)$ & 19.3 & -37.9 \\
\hline TS/IM3a (TD-O) & 19.3 & -44.5 \\
\hline TS/IM3b (TD- $\square)$ & 18.6 & -43.9 \\
\hline TS/IM4 (CH $\left.{ }_{4}-\diamond\right)$ & 28.8 & -10.8 \\
\hline
\end{tabular}

b) Solvent calculations: $\mathrm{RuO}_{4}$ (transition states only / left: aceton / right: acetonitrile)

\begin{tabular}{|l|l|l|}
\hline & $\Delta E_{\text {calc }}^{\neq_{\text {cal }}}$ & $\Delta E_{\text {calc }}^{\neq^{A N}}$ \\
\hline TS1a(AD-O) & 14.1 & 13.7 \\
\hline TS1b (AD- $\square$ ) & 16.8 & 16.6 \\
\hline TS2a (CD-O) & 14.6 & 14.6 \\
\hline TS2b (CD- $\square)$ & 16.7 & 16.4 \\
\hline TS3a (TD-O) & 17.7 & 17.7 \\
\hline TS3b (TD- $\square)$ & 16.1 & 16.2 \\
\hline
\end{tabular}


c) Gas-phase: $\mathrm{RuO}_{4}(\mathrm{OH})^{-}$

\begin{tabular}{|l|l|l|}
\hline & $\Delta E_{\text {calc }}^{\text {gas }}$ & $\Delta E_{\text {calc }}^{I M}$ \\
\hline TS/IM1a(AD-O) & 15.5 & -62.0 \\
\hline TS/IM1b (AD- $\square)$ & 19.6 & -62.9 \\
\hline TS/IM2a (CD-O) & 13.9 & -60.8 \\
\hline TS/IM2b (CD- $\square)$ & 17.7 & -62.5 \\
\hline TS/IM3a (TD-O) & 14.6 & -59.3 \\
\hline TS/IM3b (TD- $\square)$ & 15.2 & -58.6 \\
\hline TS/IM4 (CH $\left.{ }_{4}-\diamond\right)$ & 21.5 & -52.9 \\
\hline
\end{tabular}

\section{3) Plots of the intermediates}

$\operatorname{IM1a}(\Delta \mathrm{G}=-35.8 \mathrm{kcal} / \mathrm{mol})$

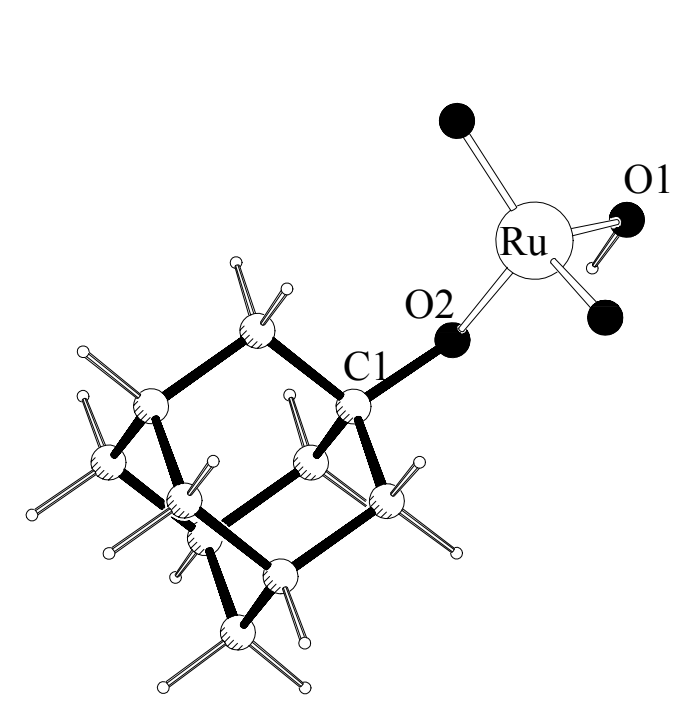

$\mathrm{Ru}-\mathrm{O} 1$ :

$\mathrm{Ru}-\mathrm{O} 2$ :

$\mathrm{O} 2-\mathrm{C} 1$ :
1.89

1.90

1.45
$\operatorname{IM1b}(\Delta \mathrm{G}=-26.7 \mathrm{kcal} / \mathrm{mol})$

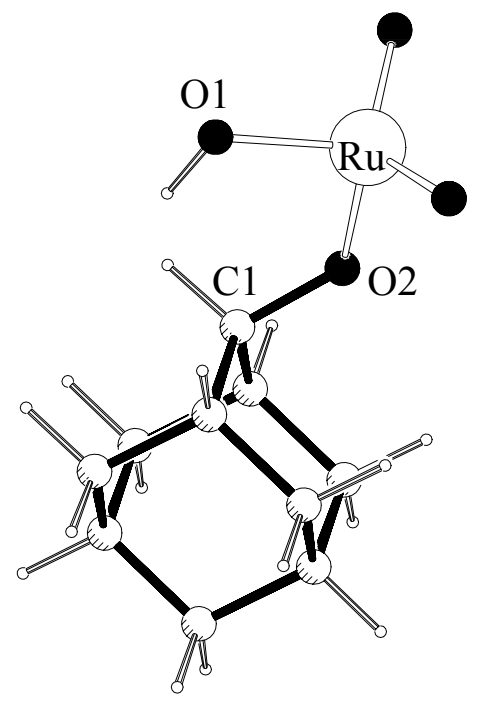
$\mathrm{Ru}-\mathrm{O} 1$ :
1.90
$\mathrm{Ru}-\mathrm{O} 2$ :
1.90
$\mathrm{O} 2-\mathrm{C} 1$ :
1.43 
$\operatorname{IM} 2 \mathrm{a}(\Delta \mathrm{G}=-25.8 \mathrm{kcal} / \mathrm{mol})$

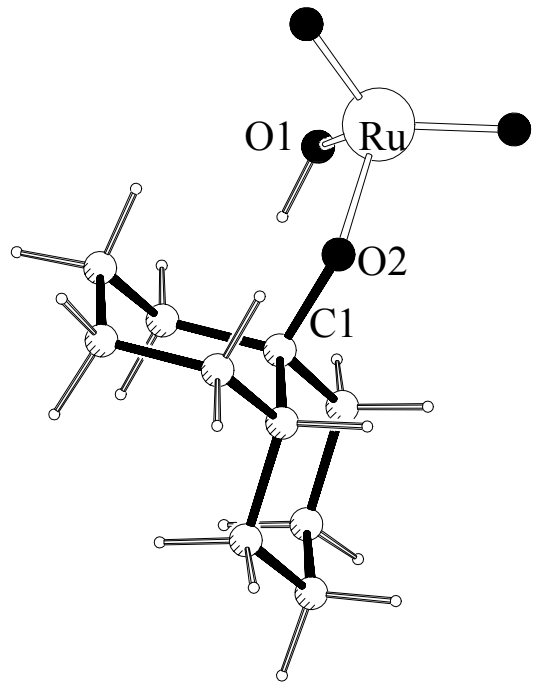

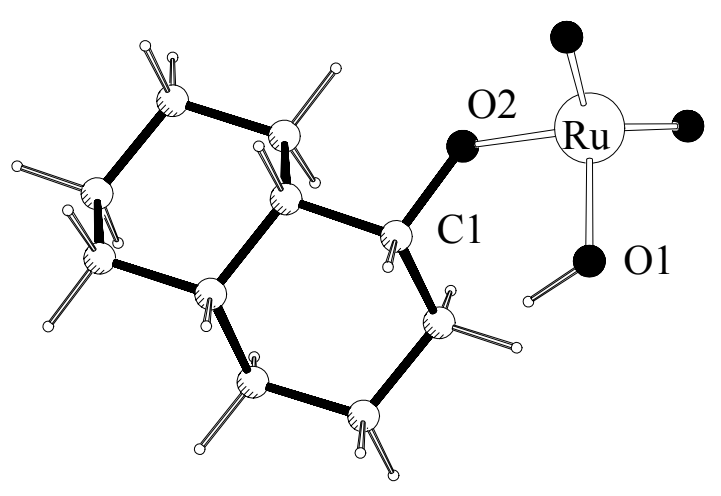

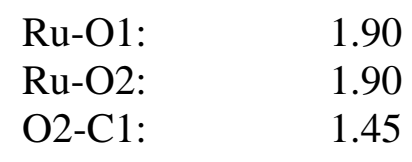

$\operatorname{IM} 3 \mathrm{a}(\Delta \mathrm{G}=-32.4 \mathrm{kcal} / \mathrm{mol})$

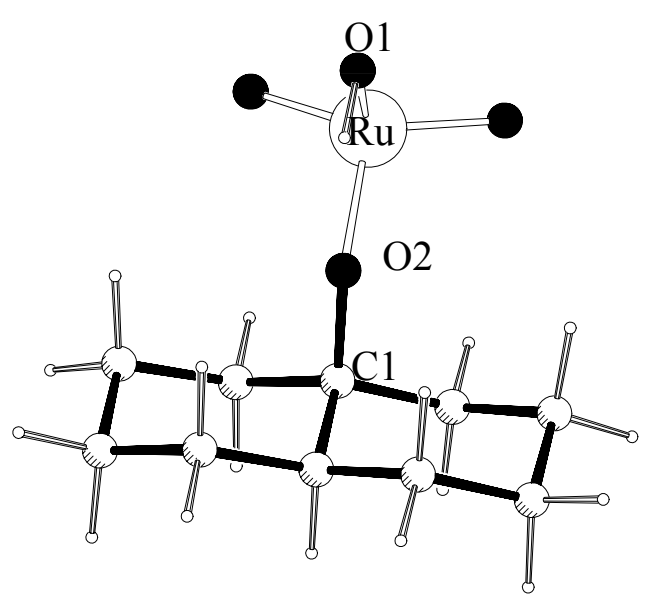
$\mathrm{Ru}-\mathrm{O} 1:$
1.90
$\mathrm{Ru}-\mathrm{O} 2$ :
O2-C1:
1.90
1.43

$\operatorname{IM} 3 \mathrm{~b}(\Delta \mathrm{G}=-32.1 \mathrm{kcal} / \mathrm{mol})$

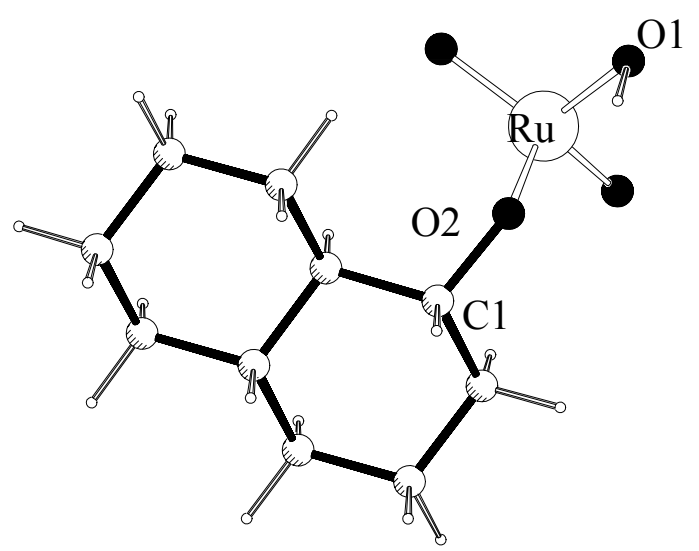

Ru-O1: $\quad 1.89$

Ru-O2: $\quad 1.90$

O2-C1: $\quad 1.45$ 
IM5a $(\Delta \mathrm{G}=-47.4 \mathrm{kcal} / \mathrm{mol})$

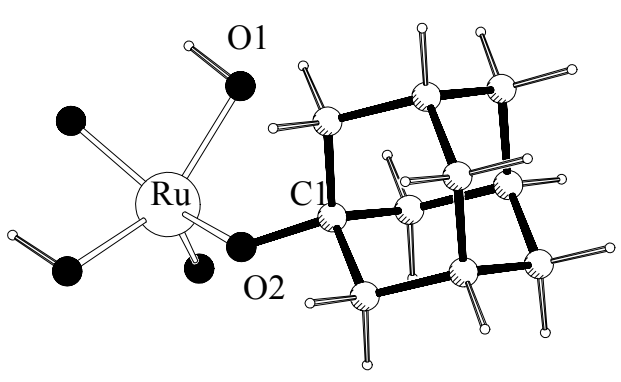

$\mathrm{Ru}-\mathrm{O} 1$ :

$\mathrm{Ru}-\mathrm{O} 2$ :

$\mathrm{O} 2-\mathrm{C} 1$ :

1.93

1.98

1.41
IM5b $(\mathrm{G}=-48.8 \mathrm{kcal} / \mathrm{mol})$

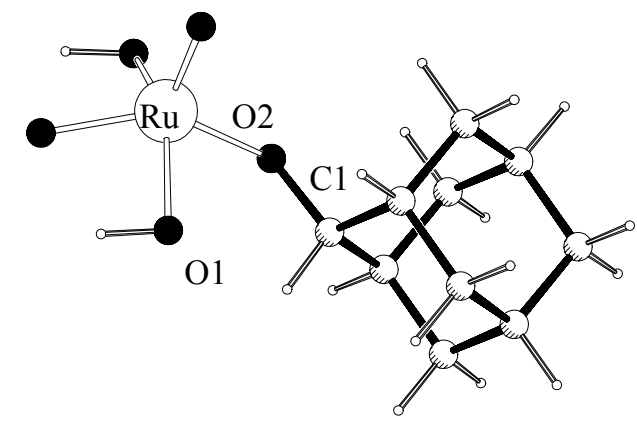
$\mathrm{Ru}-\mathrm{O} 1:$
1.93
$\mathrm{Ru}-\mathrm{O} 2$ :
$\mathrm{O} 2-\mathrm{C} 1$ :
1.98
1.41

IM6a $(\Delta \mathrm{G}=-45.9 \mathrm{kcal} / \mathrm{mol})$

$\operatorname{IM6b}(\Delta \mathrm{G}=-47.7 \mathrm{kcal} / \mathrm{mol})$
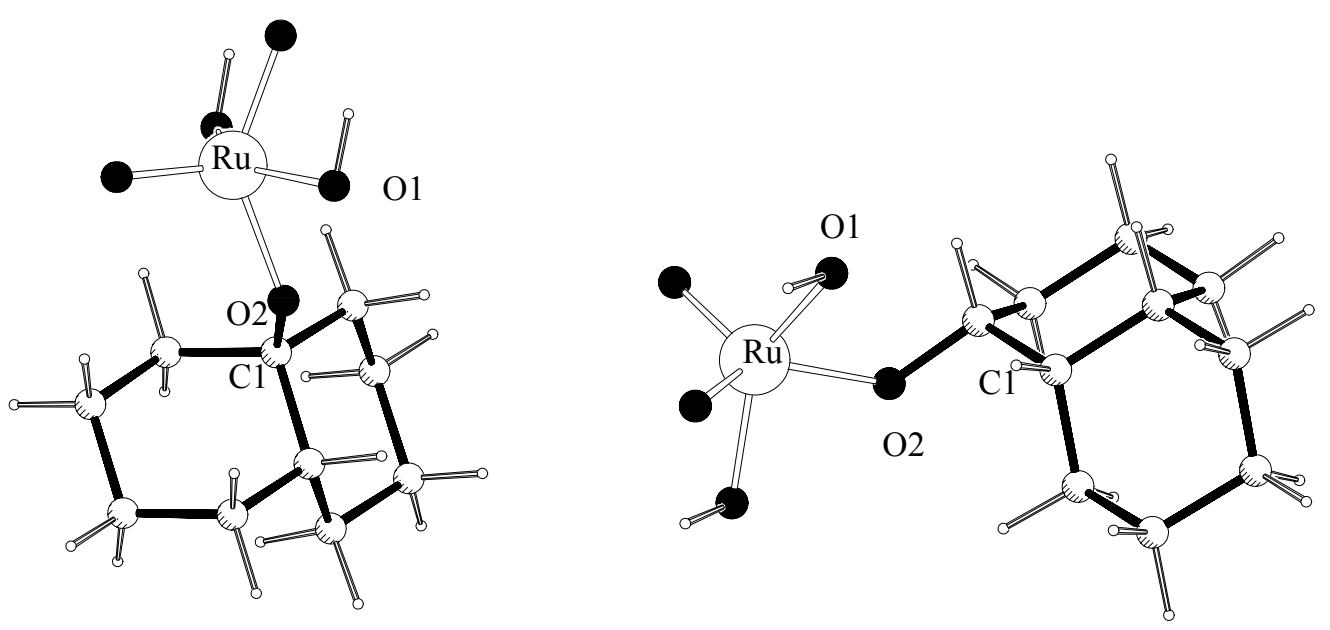
$\mathrm{Ru}-\mathrm{O} 1$ :
1.93
$\mathrm{Ru}-\mathrm{O} 2$ :
1.98
$\mathrm{O} 2-\mathrm{C} 1$ :
1.42

Ru-O1:

1.93

Ru-O2: $\quad 1.97$

O2-C1: $\quad 1.41$ 
$\operatorname{IM} 7 \mathrm{a}(\Delta \mathrm{G}=-46.3 \mathrm{kcal} / \mathrm{mol})$

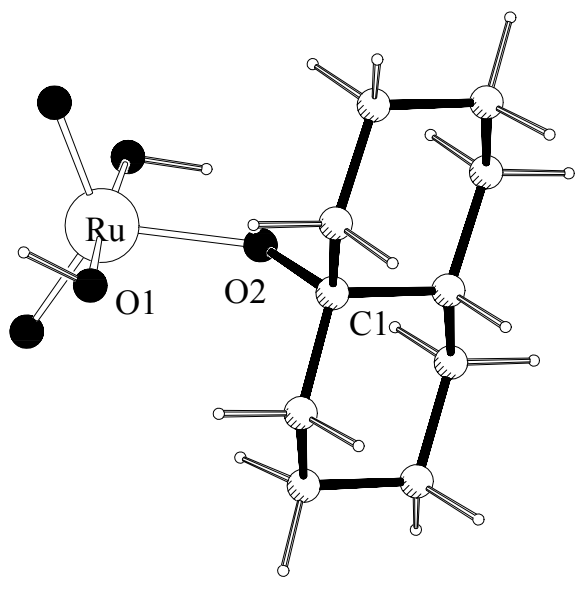

$\operatorname{IM} 7 \mathrm{~b}(\Delta \mathrm{G}=-45.5 \mathrm{kcal} / \mathrm{mol})$

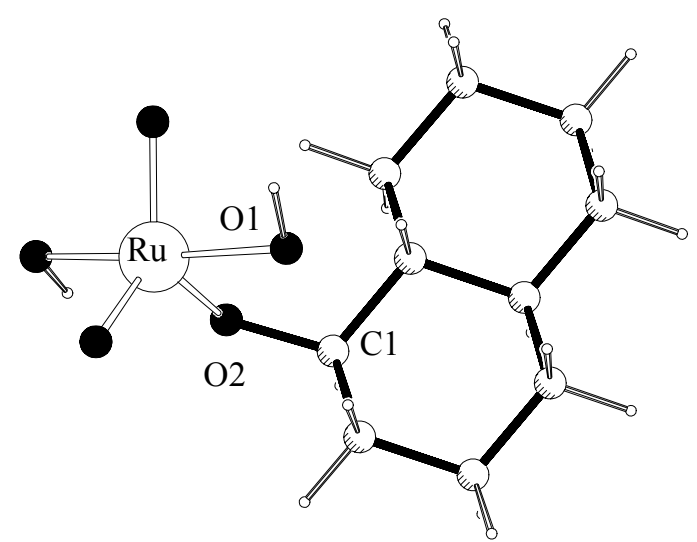

Ru-O1: $\quad 1.99$

$\mathrm{Ru}-\mathrm{O} 2: \quad 1.95$

O2-C1: $\quad 1.42$ 